\title{
Human prostate cancer metastases target the hematopoietic stem cell niche to establish footholds in mouse bone marrow
}

\author{
Yusuke Shiozawa, ${ }^{1}$ Elisabeth A. Pedersen, ${ }^{1}$ Aaron M. Havens, ${ }^{1,2}$ Younghun Jung, ${ }^{1}$ \\ Anjali Mishra, ${ }^{1}$ Jeena Joseph, ${ }^{1}$ Jin Koo Kim, ${ }^{1}$ Lalit R. Patel, ${ }^{3}$ Chi Ying, ${ }^{3}$ \\ Anne M. Ziegler, ${ }^{1}$ Michael J. Pienta, ${ }^{1}$ Junhui Song, ${ }^{4}$ Jingcheng Wang, ${ }^{1}$ \\ Robert D. Loberg, ${ }^{3}$ Paul H. Krebsbach, ${ }^{4}$ Kenneth J. Pienta, ${ }^{3}$ and Russell S. Taichman ${ }^{1}$

\begin{abstract}
${ }^{1}$ Department of Periodontics and Oral Medicine, University of Michigan School of Dentistry, Ann Arbor, Michigan, USA. ${ }^{2}$ Harvard School of Dental Medicine, Boston, Massachusetts, USA. '3epartment of Urology and Department of Internal Medicine, University of Michigan Medical School,

Ann Arbor, Michigan, USA. ${ }^{4}$ Department of Biologic and Materials Sciences, University of Michigan School of Dentistry, Ann Arbor, Michigan, USA
\end{abstract}

\begin{abstract}
HSC homing, quiescence, and self-renewal depend on the bone marrow HSC niche. A large proportion of solid tumor metastases are bone metastases, known to usurp HSC homing pathways to establish footholds in the bone marrow. However, it is not clear whether tumors target the HSC niche during metastasis. Here we have shown in a mouse model of metastasis that human prostate cancer (PCa) cells directly compete with HSCs for occupancy of the mouse HSC niche. Importantly, increasing the niche size promoted metastasis, whereas decreasing the niche size compromised dissemination. Furthermore, disseminated PCa cells could be mobilized out of the niche and back into the circulation using HSC mobilization protocols. Finally, once in the niche, tumor cells reduced HSC numbers by driving their terminal differentiation. These data provide what we believe to be the first evidence that the HSC niche serves as a direct target for PCa during dissemination and plays a central role in bone metastases. Our work may lead to better understanding of the molecular events involved in bone metastases and new therapeutic avenues for an incurable disease.
\end{abstract}

\section{Introduction}

Metastases represent the most common malignant tumors involving the skeleton: nearly $70 \%$ of patients with breast cancer or prostate cancer (PCa) - and approximately $15 \%-30 \%$ of patients with carcinomas of the lung, colon, stomach, bladder, uterus, rectum, thyroid, or kidney - have bone lesions (1). Several mechanisms are thought to account for the organ-specific nature of bone metastases, including direct tumor extensions, retrograde venous flow, and tumor embolization. It is also clear, however, that anatomy alone does not explain the organ-specific pattern of metastasis.

One hypothesis that has gained favor is that the metastatic process is functionally similar to the homing behavior of HSCs to the $\operatorname{BM}(2,3)$. HSC homing, quiescence, and self-renewal in the BM are now known to depend on a region termed the HSC niche $(4,5)$. Recent studies identified cells of the osteoblastic and endothelial lineages as key components of the niche (6-11). Molecules that play critical roles in HSC niche selection are now thought to be used by metastases to establish footholds in the $\operatorname{BM}(2,3)$, including chemoattractants (CXCL12; also referred to as stromal-derived factor-1; refs. 3, 12), attachment factors (annexin II [Anxa2]; ref. 13), regulators of cell growth, and vascular recruitment (IL-6 and VEGF; ref. 14). Once in the BM, tumor cells parasitize the bone microenvironment to regulate long-term survival/dormancy and, ultimately, metastatic growth. However, it is not known whether metastatic cells specifically target the HSC niche during dissemination.

In the present work, we used a PCa model to demonstrate that tumors directly compete with HSCs for occupancy of the endosteal HSC niche during BM transplantation (BMT). Critically, HSCs

Conflict of interest: The authors have declared that no conflict of interest exists. Citation for this article: J Clin Invest. 2011;121(4):1298-1312. doi:10.1172/JCI43414. colocalized with PCa cells to the endosteal bone surfaces in vivo and in vitro, suggestive of niche competition. Additional evidence suggesting that PCa cells target the HSC niche during metastases was obtained when the osteoblastic niche was ablated using conditional osteoblast knockout tissues (Col2.3L-TK; ref. 15) in metastatic assays. Our results showed that fewer metastatic cells homed to the BM when the HSC niche was compromised. Conversely, increasing the number of HSC niches with parathyroid hormone (PTH) promoted metastasis. Once in the niche, metastatic cells like HSCs could be mobilized back into the peripheral blood using agents that mobilize HSCs. Importantly, disseminated PCa cells reduced the number of HSCs in the BM by driving HSCs into progenitor pools and peripheral blood. These findings demonstrated that PCa cells and HSCs compete for the endosteal HSC niche and use the same mechanisms to access and egress the niche. These data provide direct evidence that the endosteal HSC niche plays a central role in bone metastases.

\section{Results}

$P C$ a cells target the HSC niche during metastasis. To directly test whether metastatic cells compete with HSCs for the niche, disseminated PCa cells were evaluated for their ability to prevent HSC engraftment. We used a micrometastasis model to determine whether PCa cells target the HSC niche during metastasis to bone (Supplemental Methods; supplemental material available online with this article; doi:10.1172/JCI43414DS1). In this model, disseminated tumor cells shed from s.c. implanted tumors, which can be tracked by quantitative real-time PCR (QPCR), have the capacity to generate metastatic lesions over time in the BM (16). For these studies, to establish disseminated PCa cells in the niche, NOD/SCID mice (CD45.1) were implanted s.c. with PCa tumors or with nonmeta- 
A s.c. $\mathrm{PCa}$

or

s.c. NMPE

or

Sham Surgery Tumor Removal

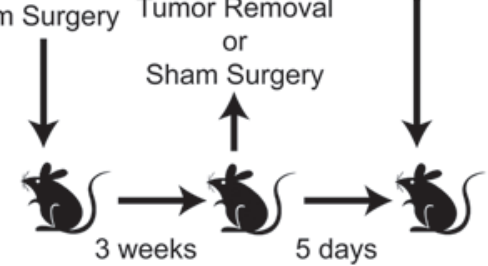

NOD/SCID

CD45.1

\section{C}
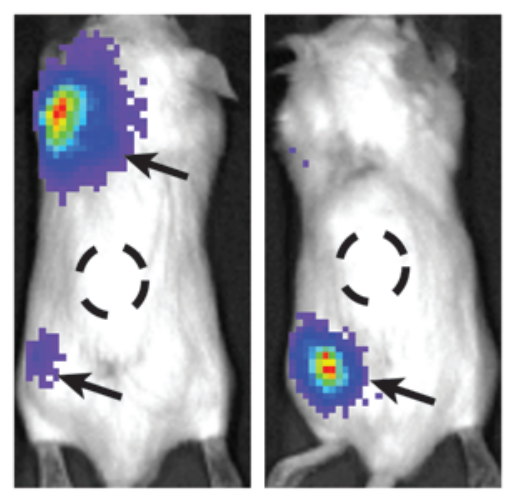

B $\longrightarrow$ Surgical Control

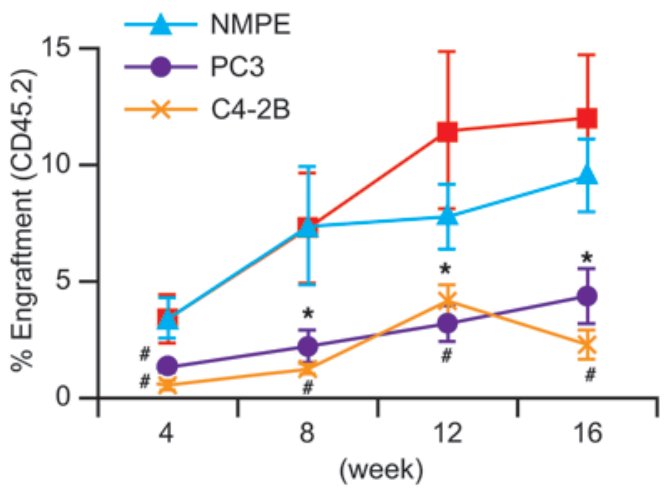

D
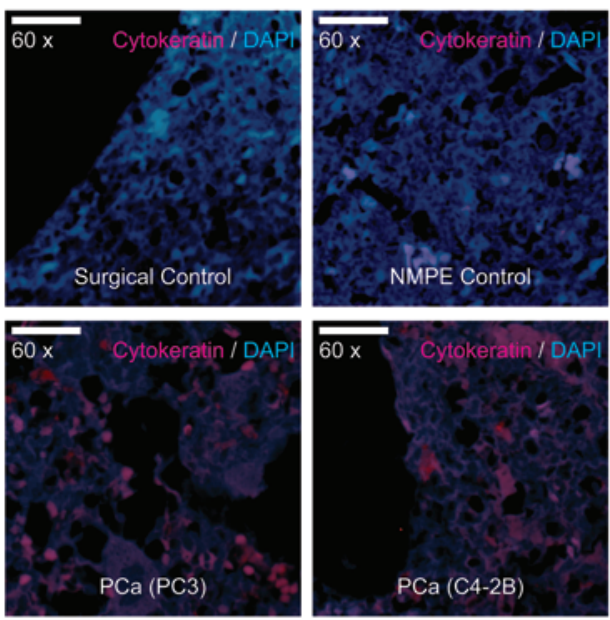

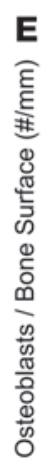
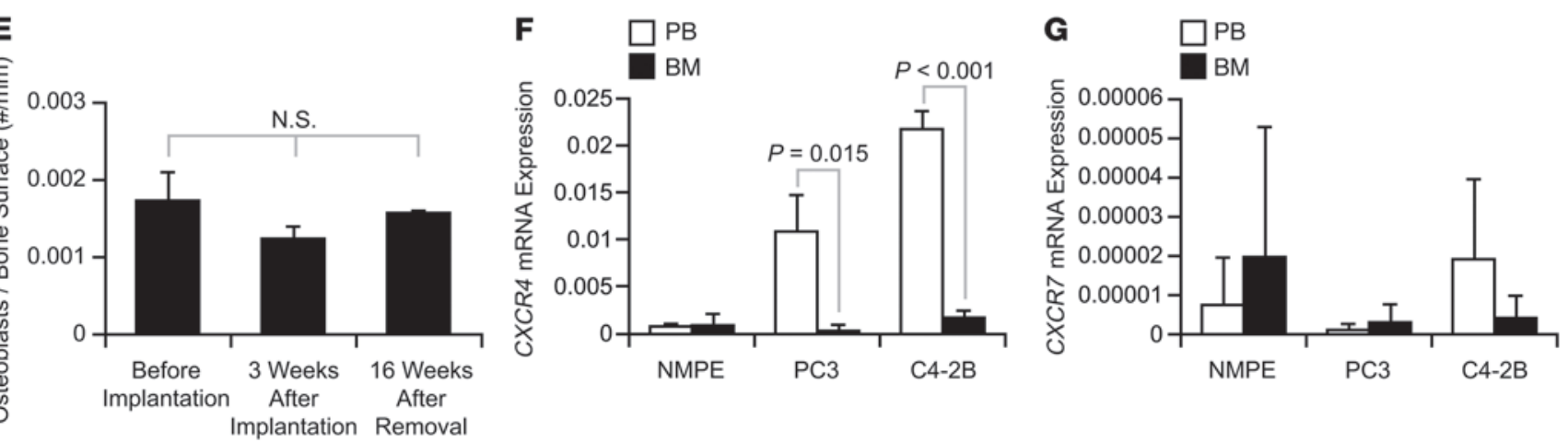

Figure 1

PCa cells compete for the HSC niche and prevent HSC engraftment. (A) Experimental model of BMT in the presence or absence of disseminated PCa cells. (B) Fewer donor HSCs (CD45.2) were able to engraft into NOD/SCID mice (CD45.1) when disseminated tumor cells (PC3 or C4-2B PCa cells) were present in BM. ${ }^{*} P<0.05,{ }^{\#} P<0.01$ versus NMPE, Student's $t$ test ( $n=10$ per group). (C) At $4,8,12$, and 16 weeks after transplantation, the establishment of metastases was followed by bioluminescent imaging. Data are representative of bioluminescent imaging of mice that had developed metastases at approximately 40 weeks. Dashed circles denote where the primary s.c. tumors were implanted and removed. Arrows show metastatic PCa. (D) Representative images of BM histology of mice that developed micrometastases at 16 weeks. Original magnification, $\times 60$. Scale bars: $50 \mu \mathrm{m}$. (E) Osteoblast numbers were determined in the long bones. $(\mathbf{F}$ and $\mathbf{G}) \mathrm{mRNA}$ levels of $(\mathbf{F})$ CXCR4 and $(\mathbf{G})$ CXCR7 in PCa cells at peripheral blood (PB) and BM. Significance of differences was determined by Kruskal-Wallis test.

static transformed prostate epithelial (NMPE) cell lines as controls (Figure 1A and Supplemental Methods). Later, the tumors were removed, and BMT was performed using BM cells derived from CD45.2 animals (Figure 1A). To preserve the integrity of the niche, preparative transplant regimens (e.g., radiation and chemotherapy) were not used, and engraftment was analyzed by FACS. In all cases, greater HSC engraftment was observed in control groups (NMPE or no tumor implanted) than in the tumor-bearing animals (Figure $1 \mathrm{~B}$ ), which suggests that the cells shed from a primary tumor prevent HSC engraftment by occupying the HSC niche. 
A

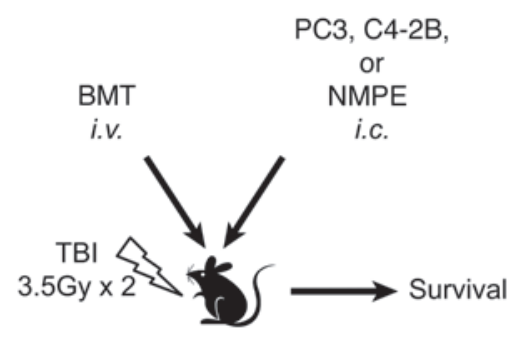

C
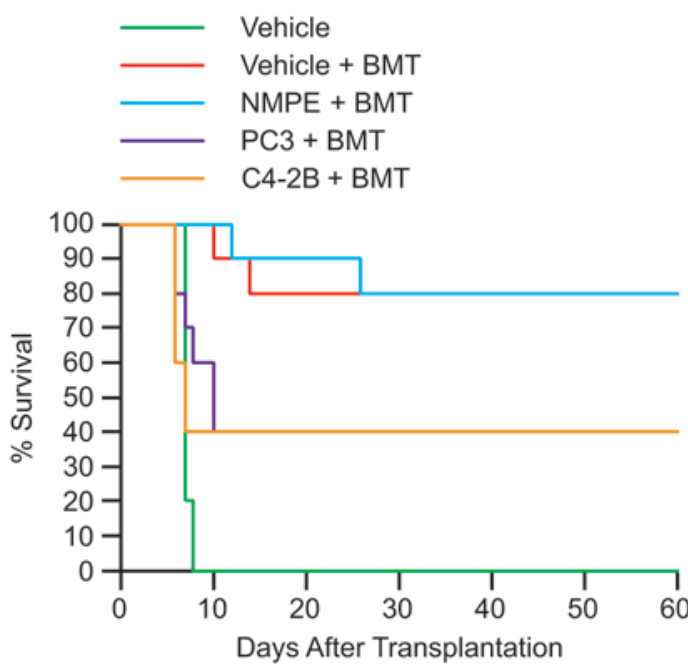

$\mathbf{E}$

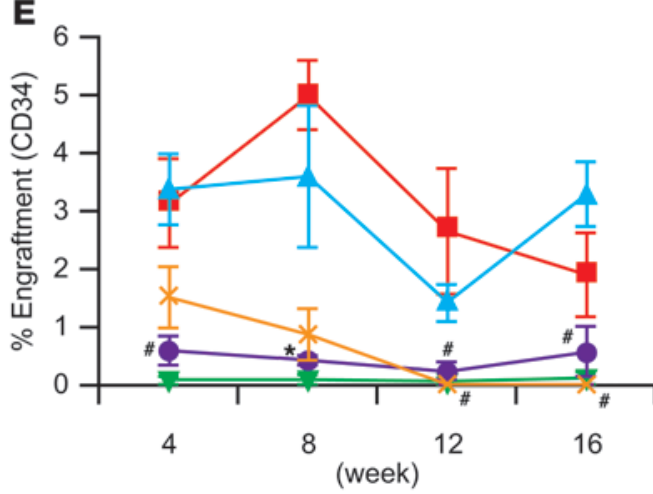

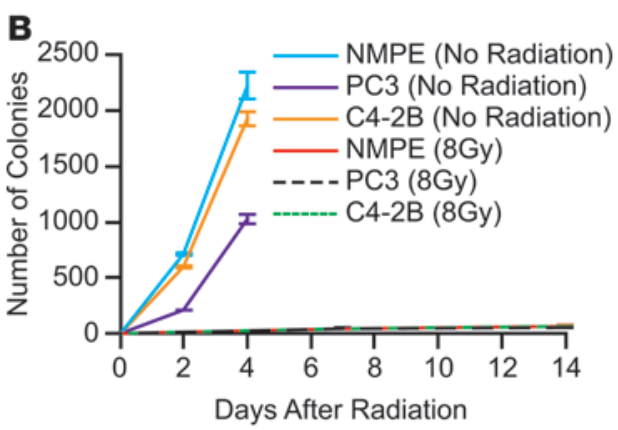

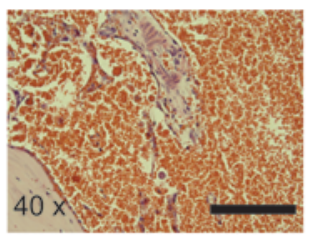

Vehicle

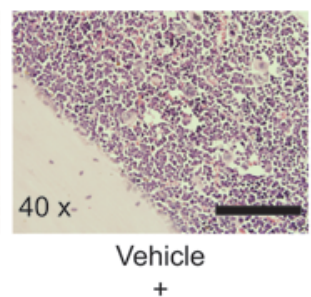

BMT
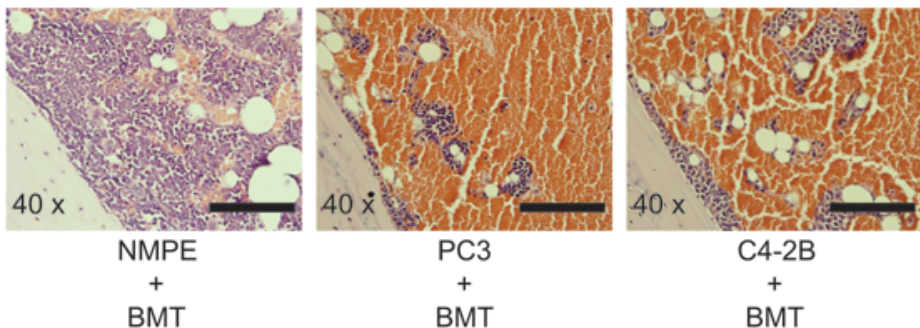

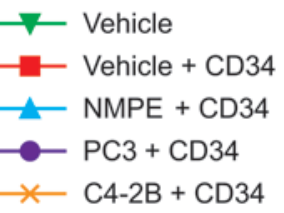

\section{Figure 2}

Direct competition for the HSC niche between HSCs and PCa cells. (A) Competitive BMT experimental model. BMT was performed in the presence or absence of PC3 or C4-2B PCa cells or NMPE control cells. TBI, total body irradiation. (B) To avoid PCa cell proliferation in vivo, NMPE control cells or PCa cells were irradiated with 8 Gy (2x 4 Gy), and the effects of irradiation on the ability of 5,000 PCa cells to form colonies of 50 cells or greater were analyzed. Nonirradiated cells served as positive controls. Irradiation inhibited the colony-forming abilities of NMPE cells and PCa cells. (C) Kaplan-Meier survival plots after BMT in the presence or absence of PC3 or C4-2B PCa cells or NMPE control cells. Survival was monitored up to 60 days. $P=0.038$, PC3 versus NMPE; $P=0.041, C 4-2 B$ versus NMPE, log-rank test $(n=10$ per group). (D) Representative $\mathrm{BM}$ histology after competitive BMT of animals in C. Original magnification, $\times 40$. Scale bars: $50 \mu \mathrm{m}$. (E) Engraftment of human BM CD34+ cells into NOD/SCID I/2 $\mathrm{rg}^{-/-}$mice in the presence or absence of PC3 or C4-2B PCa cells or NMPE control cells. ${ }^{*} P<0.05$, ${ }^{\#} P<0.01$ versus NMPE ( $n=10$ per group, Student's $t$ test). Human PCa cells prevented human HSC engraftment.

To verify that the reduced HSC engraftment was in fact caused by the disseminated PCa cells, we used bioluminescent imaging and observed that tumors developed from the metastatic cells in these animals ( 4 weeks, 0 of 10; 8 weeks, 0 of 10; 12 weeks, 2 of 10; 16 weeks, 3 of 10; Figure 1C). Additionally, disseminated PCa cells in the BM were identified by immunohistochemistry; few or no disseminated cells were observed in either of the controls (Figure 1D and Supplemental Figure 1A). Furthermore, these results were not likely attributable to changes in the number or size of the niche (e.g., osteoblast number; Figure 1E) resulting from the primary tumor alone. 
A
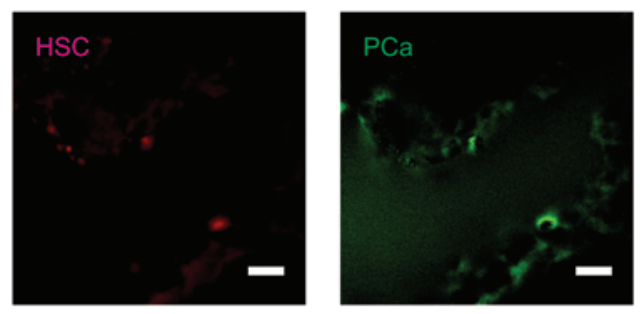

B

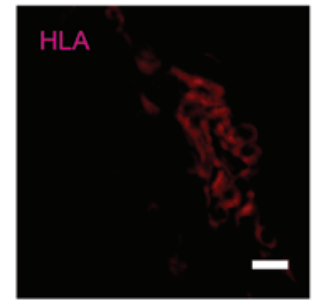

C

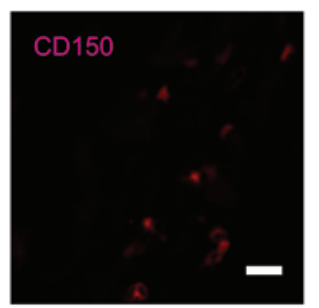

D

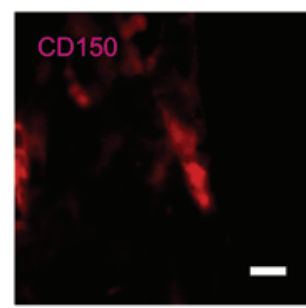

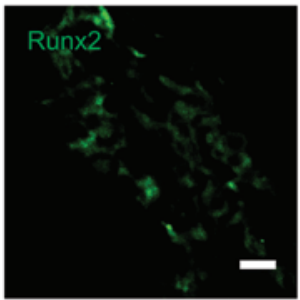
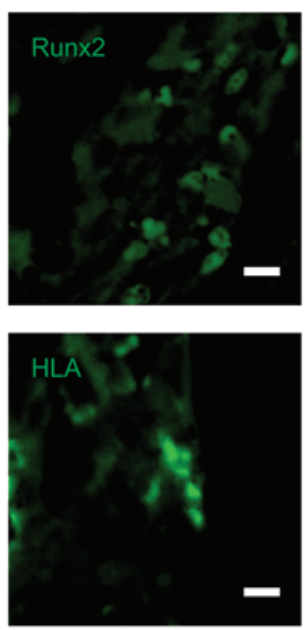
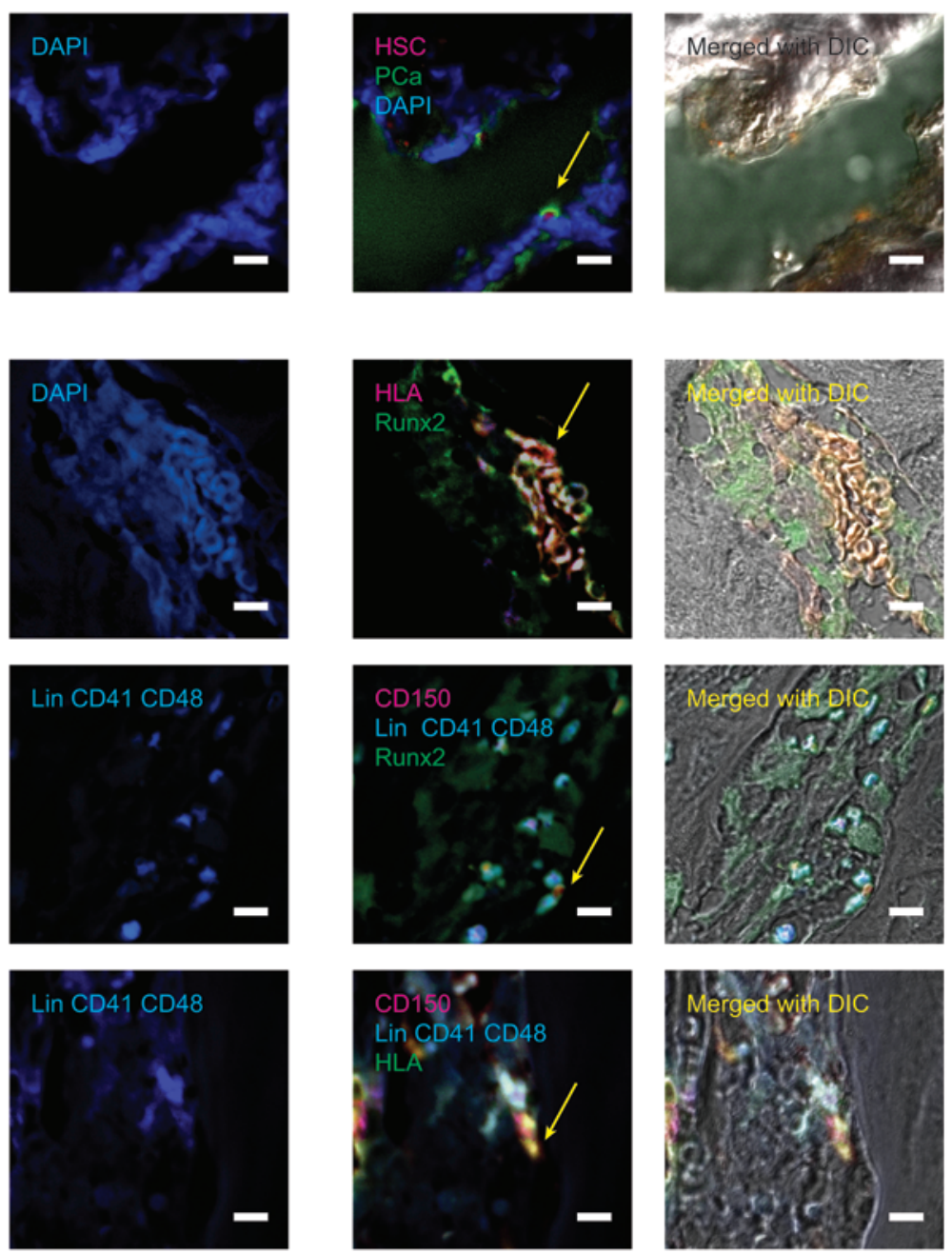
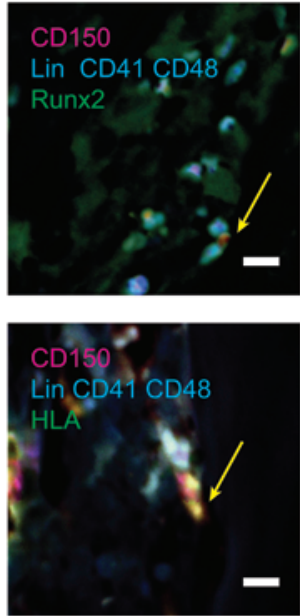
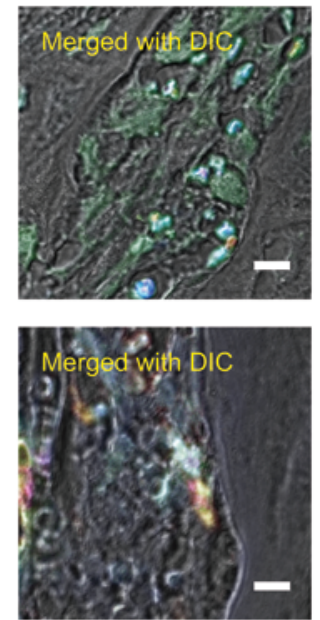

Figure 3

HSCs and PCa cells colocalize to BM niches through Runx2. (A) To determine whether metastatic cells and HSC colocalize to the same niche, a confocal microscope was used to track prelabeled LSK HSCs (red) and prelabeled PCa cells (green) 24 hours after transplantation. Nuclei were stained with DAPI (blue). DIC, differential image contrast. (B-D) SCID mice were implanted with PCa cells. After 3 weeks, the long bones were collected. Representative elements of the BM were triple-stained with (B) anti-HLA antibodies, anti-Runx2 antibodies, and DAPI; (C) antiCD150 antibodies, anti-lineage antibody cocktail, and anti-Runx2 antibodies; and (D) anti-CD150 antibodies, anti-lineage antibody cocktail, and anti-HLA antibodies. Arrows denote colocalization of HSCs and PCa cells (A and D), osteoblasts and PCa cells (B), or osteoblasts and HSCs (C). Original magnification, $\times 60$. Scale bars: $10 \mu \mathrm{m}$.

CXCL12 and its receptors, CXCR4 and CXCR7, are known to play major roles in HSC homing to the $\mathrm{BM}(17,18)$ and in establishing PCa metastases in bone $(3,12,19-21)$. To determine whether a similar mechanism regulates PCa cell targeting to the HSC niche, mRNA levels of CXCR4 and CXCR7 expressed by PCa cells in the niche were compared with those of circulating PCa cells in the blood. Compared with PCa cells in culture (Supplemental Figure 1, B and C) or in peripheral blood, CXCR4 expression was dramatically reduced in PCa cells isolated from the HSC niche, whereas no remarkable changes were observed in CXCR7 levels (Figure 1, F and G). Furthermore, time course studies tracking PCa cell dissemination into the niche along with CXCL12 BM levels (Supplemental Figure 2, A-E, and Supplemental Methods) - in conjunction with our previous work showing that CXCR4 and CXCR7 regulate metastasis $(3,12$, 19-21) - further support the concept that CXCL12 plays an important mechanism whereby PCa cells target the HSC niche.
To directly test whether disseminated tumor cells compete with HSCs for the niche, we used a competitive engraftment assay. Lethally irradiated animals were transplanted with BM cells alone or with PCa cells or NMPE control cells, and survival was evaluated as a measure of HSC engraftment (Figure 2A). To ensure that only occupancy of the niche occurred, rather than tumor growth, $\mathrm{PCa}$ and NMPE cells were irradiated to prevent proliferation (Figure 2B). Significantly more of the PCa cell-injected animals failed to engraft and subsequently required euthanasia than the control animals (Figure 2C), yet this effect was dependent on the number of PCa cells (data not shown), which suggests that PCa cells are not as efficient as HSCs in targeting the niche. BM histology demonstrated significant delays in engraftment of the animals injected with tumor cells along with their transplants (Figure 2D and Supplemental Figure 3), demonstrating competition between HSCs and PCa cells for the niche. 
A
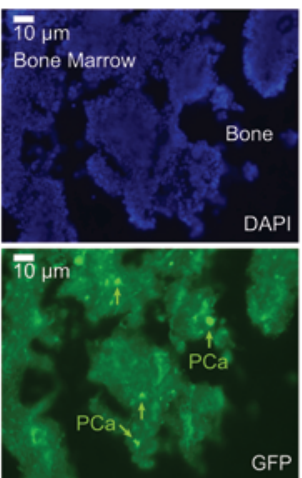

D
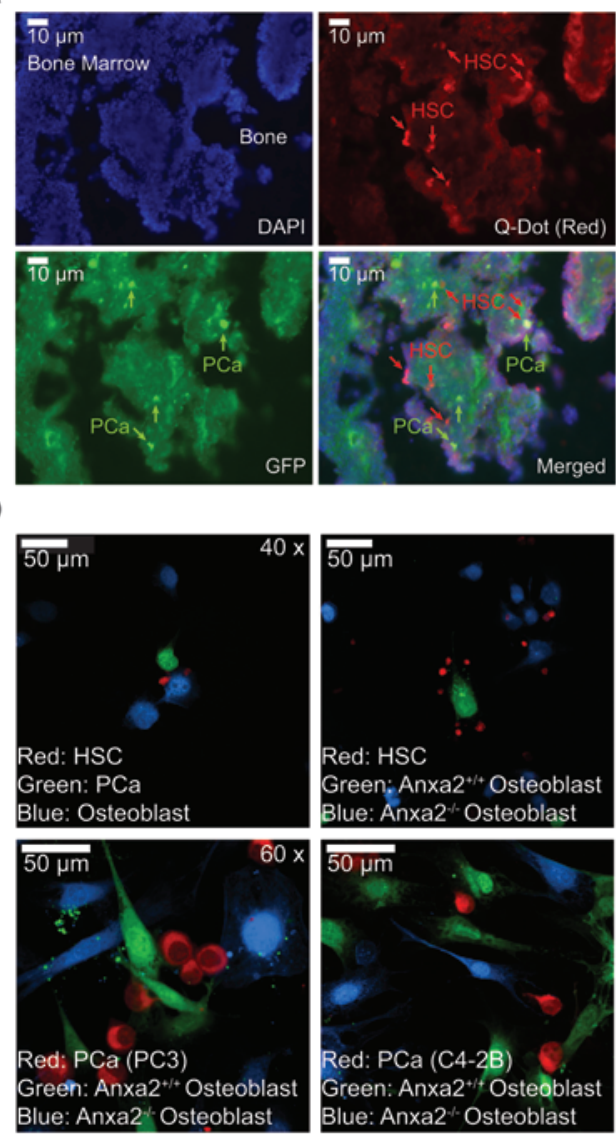

E

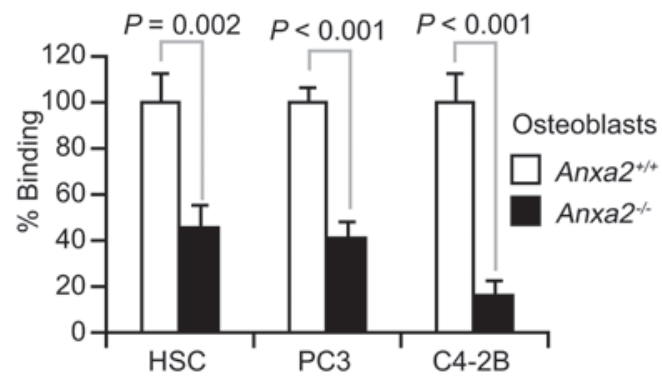

B

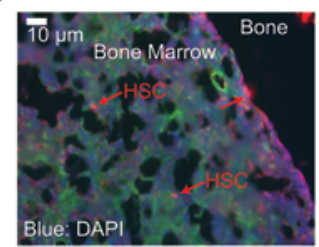

C

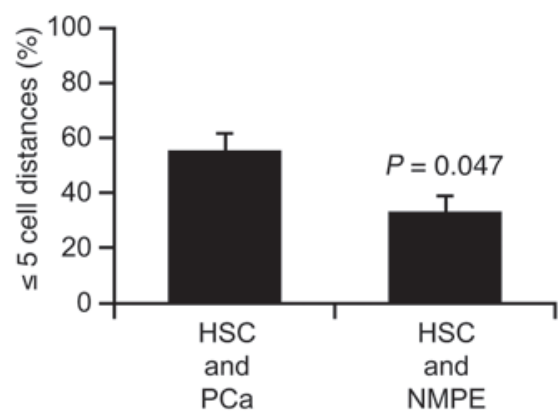

F

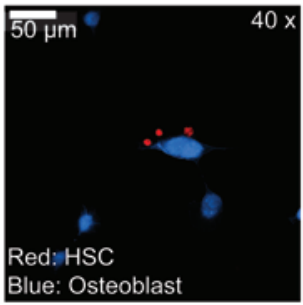

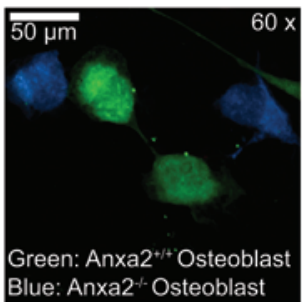

H s.c. Control or Col2.3A-TK Vossicles i.p.
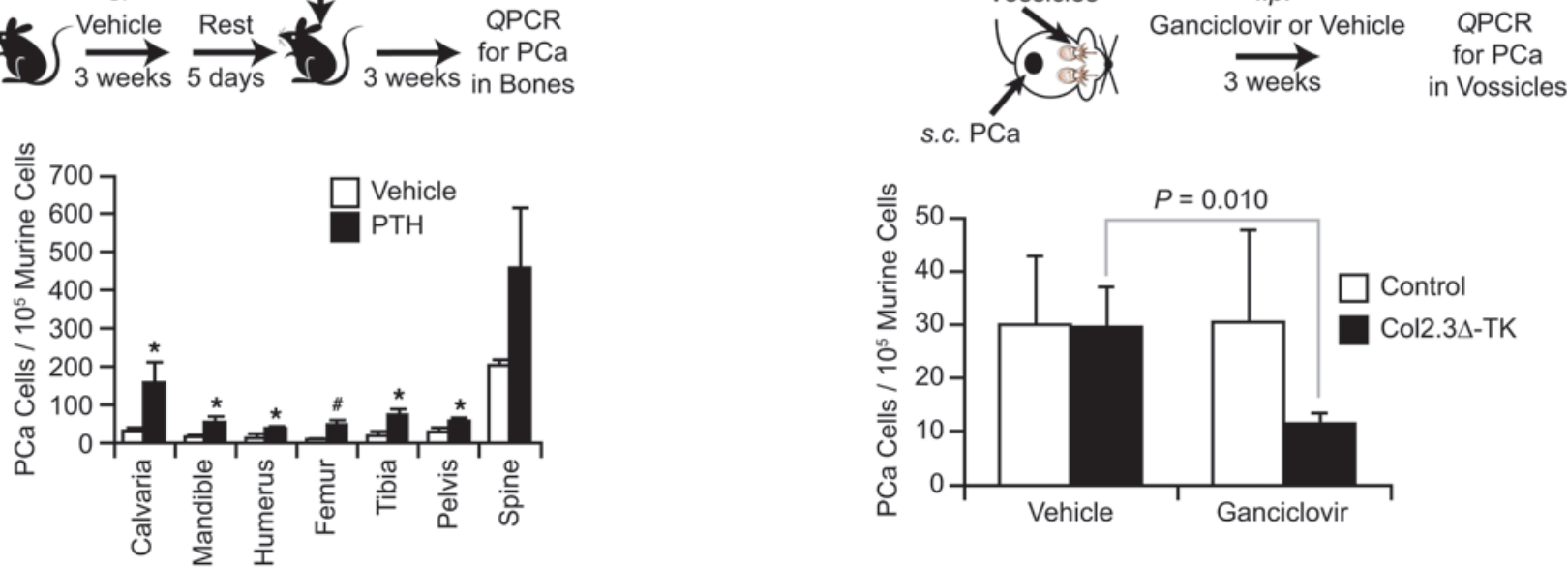
s.c. $\mathrm{PCa}$

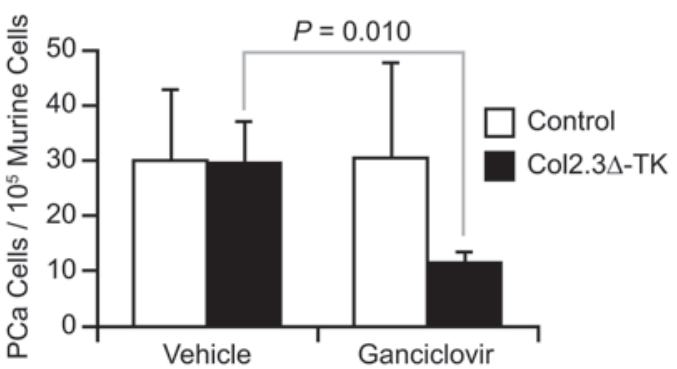

Figure 4

HSCs and PCa cells colocalize to BM niches, and alteration of niche size regulates tumor dissemination. (A and B) To determine whether metastatic cells and HSCs colocalize to the same niche, multiphoton imaging was used to track prelabeled LSK HSCs (red) and (A) prelabeled PCa cells (green) or (B) NMPE control cells 24 hours after transplantation. Nuclei were stained with DAPI (blue). Original magnification, $\times 200$. (C) Statistical analyses of A. (D) SLAM HSCs and PC3 or C4-2B PCa cells colocalized to a single osteoblast in vitro, as imaged by confocal microscopy. (E) Statistical analyses of in vitro adhesion assays to Anxa2 $2^{+/+}$versus Anxa2 ${ }^{-/}$osteoblasts (see D). (F) SLAM HSCs, but not NMPE cells, in vitro localized to a single osteoblast. NMPE cells were unable to bind to Anxa2 ${ }^{+/+}$or $A n x a 2^{-/-}$osteoblasts. (G) To expand the osteoblast numbers, animals were pretreated with vehicle or PTH prior to establishing primary tumors, and the number of metastatic PC3 cells was determined at 3 weeks ( $n=8$ per group). ${ }^{*} P<0.05,{ }^{\#} P<0.01$ versus vehicle. $(\mathrm{H})$ Homing of PC3 cells to Col2.3 $\Delta$-TK versus control vossicles with or without ganciclovir ( $n=8$ per group). The number of disseminated PCa cells homed to vehicle-treated control vossicles was set as $100 \%$. Significance of differences was determined by Student's $t$ test (C and $\mathbf{E})$ or Kruskal-Wallis test (G and $\mathbf{H})$. Scale bars: $10 \mu \mathrm{m}(\mathbf{A}$ and $\mathbf{B}) ; 50 \mu \mathrm{m}(\mathbf{D}$ and $\mathbf{F})$. 


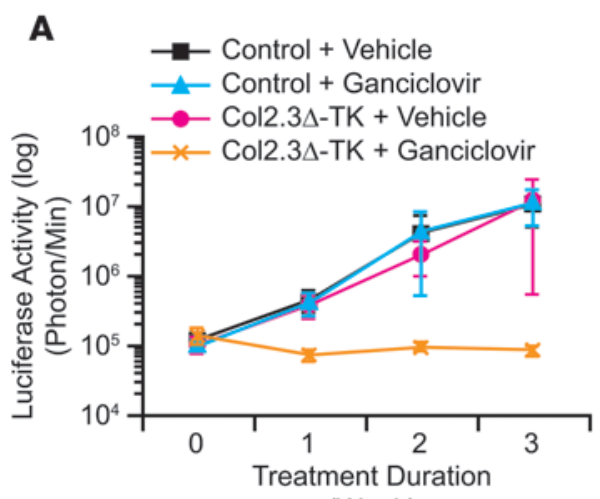

(Week)
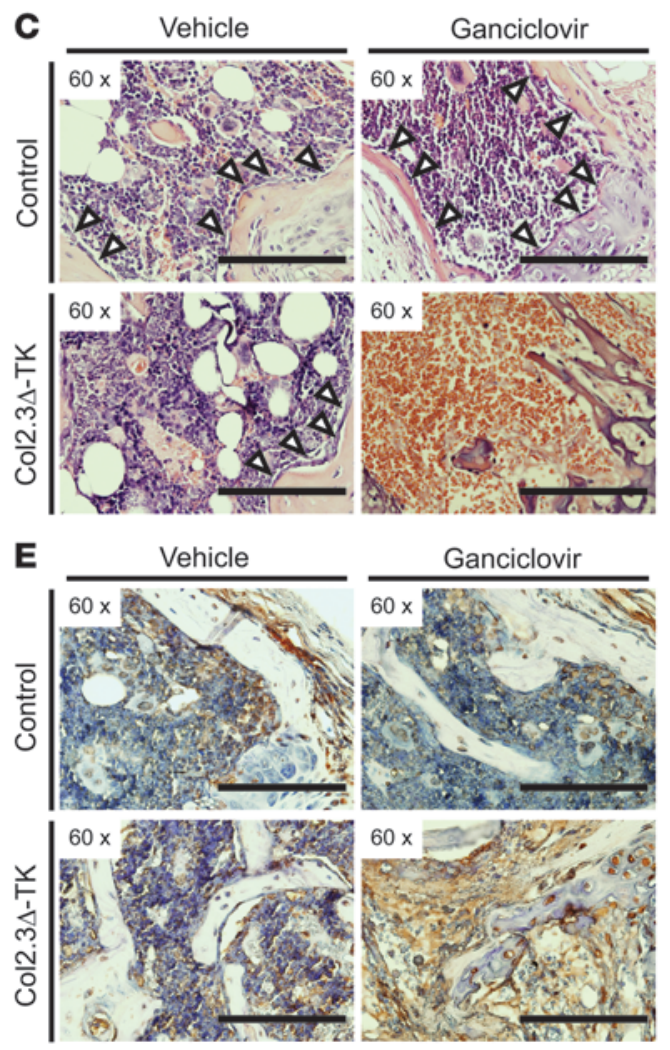

B

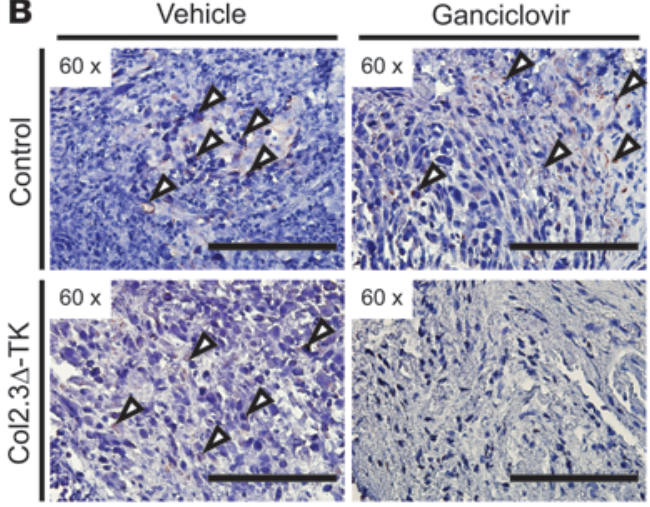

D

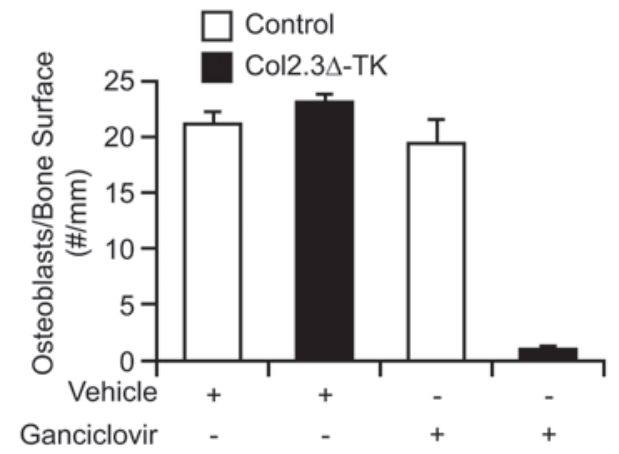

$\mathbf{F}$
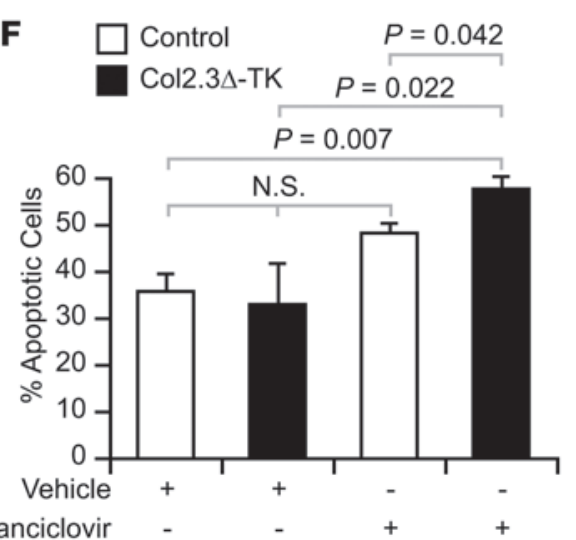

\section{Figure 5}

The osteoblastic niche is critical for PCa cell growth in bone. Luciferase-labeled PC3 cells were placed directly into Col2.3 $\Delta$-TK or control vossicles, which were subsequently implanted into the immunodeficient mice. Mice were treated with either ganciclovir or vehicle for 3 weeks to ablate the osteoblast niche. (A) Bioluminescent imaging of vossicles was performed over time, demonstrating no tumor growth in Col2.34-TK vossicles in the presence of ganciclovir ( $n=10$ per group). (B) Cytokeratin-immunostained vossicles. Arrowheads denote cytokeratin-positive cells. (C) H\&E examination of vossicles. Arrowheads denote endosteal osteoblasts. (D) Osteoblast number was quantified with H\&E staining. (E) Representative TUNEL-stained vossicles. Original magnification, $\times 60$. (F) To determine whether luciferase-labeled PC3 cells in the vossicles had undergone apoptosis, vossicles were recovered and triturated, and cells were filtered through a 40- $\mu \mathrm{m}$ cell strainer to obtain single-cell suspensions. The resulting cells were incubated first with a FITC-conjugated HLA-ABC antibody, where greater than $99 \%$ of the luciferase-labeled PC 3 cells were positively stained (not shown). Thereafter, percent apoptosis of luciferase-labeled PC3 cells in the vossicles was analyzed using PE-conjugated annexin V/ 7-ADD by gating on HLA-ABC ( $n=4$ per group). Significance of differences was determined by Student's $t$ test. Scale bars: $50 \mu \mathrm{m}$ (B, C, and E).

Thus far, our findings demonstrated that human PCa cells compete with murine HSCs for occupancy of the niche. To determine whether human PCa cells and HSCs compete for the niche in vivo, direct completion assays were performed using human $\mathrm{CD} 34^{+} \mathrm{BM}$ cells and human PCa cells in sublethally irradiated mice. Under these conditions, engraftment of the human cells was low, as expected. Sig- nificantly fewer human $\mathrm{CD} 34^{+}$cells engrafted into PCa cell-injected animals (Figure 2E). These data suggest that disseminated tumor cells directly compete with HSCs for occupancy of the niche.

PCa cells and HSCs colocalize to the endosteal niche. At present, the precise cellular composition and location of the HSC niche remains controversial $(5,22)$. Recent reports have demonstrated 
A

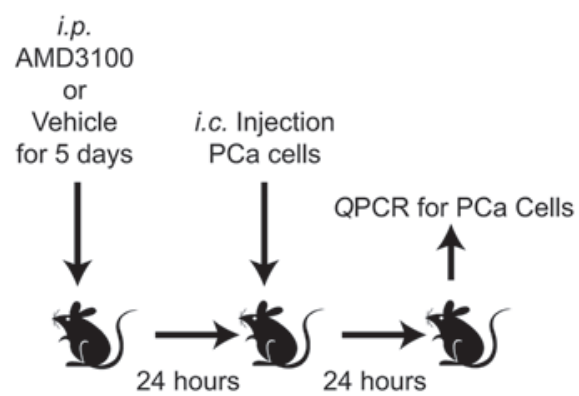

C

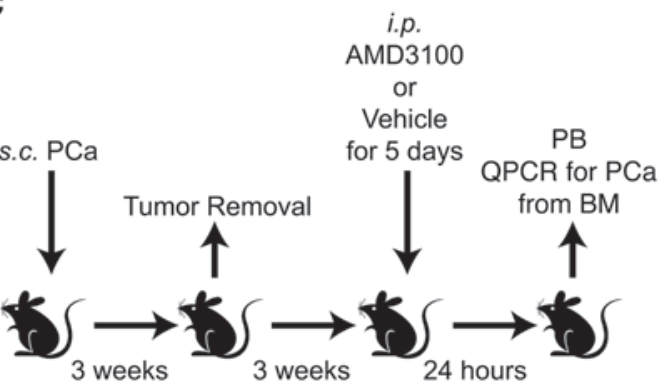

F
B

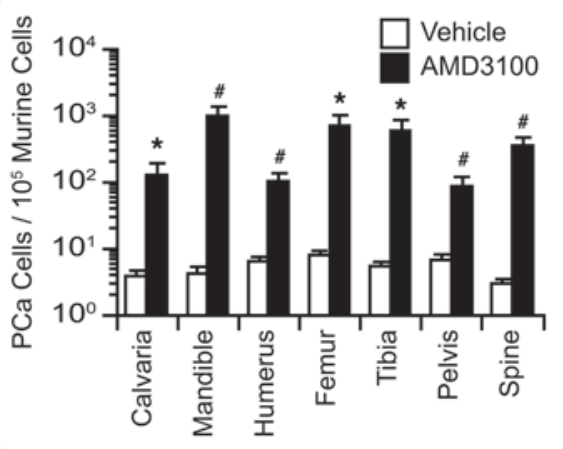

D

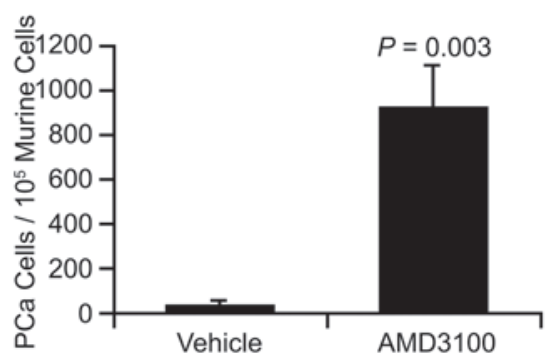

E
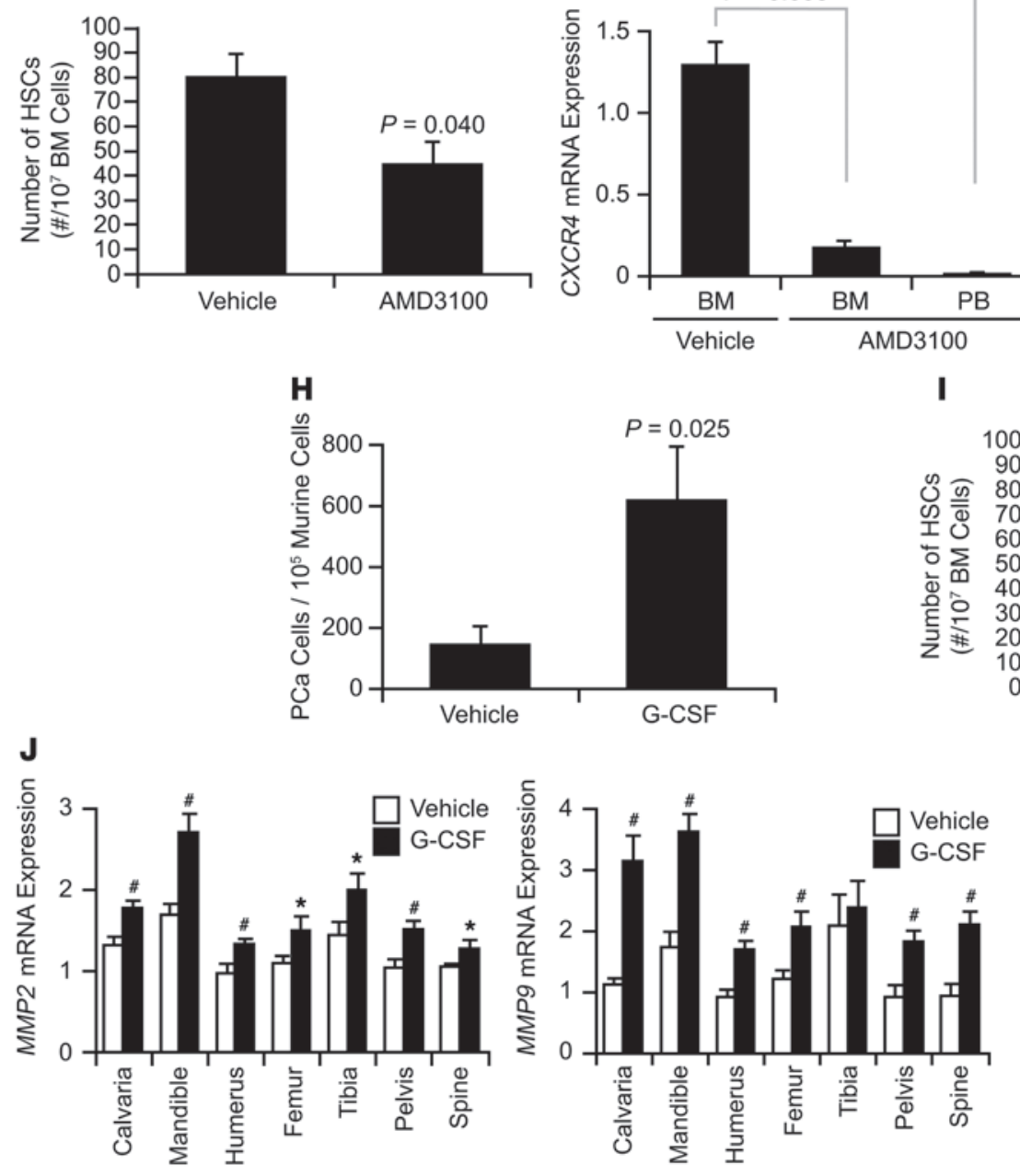

G

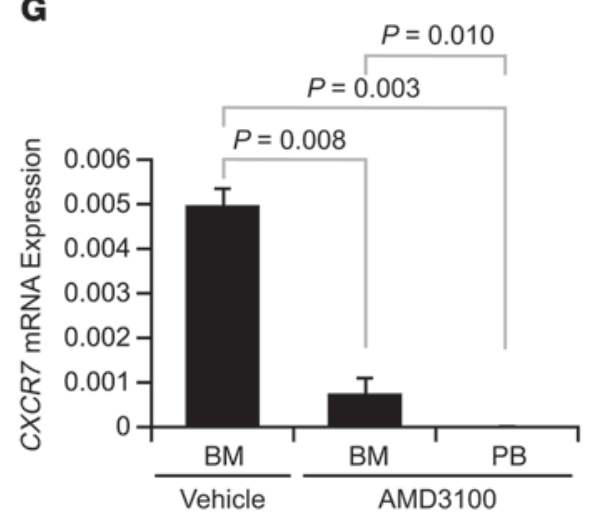

K

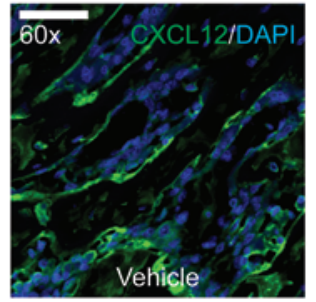

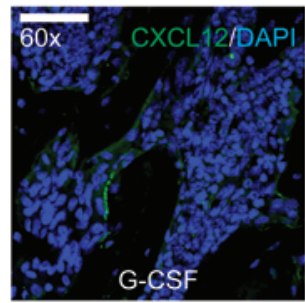




\section{Figure 6}

PCa cells target the HSC niche, and disseminated PCa cells can be mobilized from the BM niche via the CXCR4/CXCL12 axis. (A) Experimental model of HSC mobilization out of the niche via AMD3100 treatment to open the HSC niche. (B) PCa cell number in BM after i.c. injection of $1 \times 10^{6}$ cells after AMD3100 mobilization of HSCs. ${ }^{*} P<0.05$, $\# P<0.01$ versus vehicle. (C) Experimental model to determine whether AMD3100 mobilizes disseminated PCa cells from BM ( $n=8$ per group). (D) Peripheral blood levels of PC3 cells mobilized with AMD3100 or vehicle, evaluated by QPCR. (E) SLAM HSC numbers in the BM after AMD3100 treatment were enumerated by FACS. ( $F$ and $\mathbf{G}$ ) mRNA levels of (F) CXCR4 and (G) CXCR7 in HSCs at peripheral blood and BM with or without AMD3100 treatment. (H) Peripheral blood levels of PC3 cells mobilized with G-CSF or vehicle, evaluated by QPCR. (I) Number of HSCs in BM after G-CSF treatment, enumerated by FACS. (J) BM mRNA levels of MMP2 and MMP9 after G-CSF treatment, determined by QPCR. ${ }^{*} P<0.05,{ }^{\#} P<0.01$ versus vehicle. (K) CXCL12-immunostained BM. Original magnification, $\times 60$. Scale bars: $50 \mu \mathrm{m}$. Significance of differences was determined by Kruskal-Wallis test (B, D, F-H, and $\mathbf{J})$ or Student's $t$ test (E and I).

that endosteal cells of the osteoblastic lineage contribute to the development of the HSC niche (6-10). To determine whether disseminated PCa cells and HSCs colocalize to the endosteal niche, prelabeled HSCs ( $\mathrm{Lin}^{-} \mathrm{Sca}-1^{+} \mathrm{C}-\mathrm{Kit}^{+}$, referred to herein as LSK HSCs) and prelabeled PCa cells were injected into animals simultaneously. After 24 hours, the long bones were recovered, and confocal microscopy was used to track PCa cells and HSCs after transplantation. Both cell types colocalized within a few microns of each other in the BM of recipient animals (Figure 3A).

To further characterize the interactions between PCa cells and HSCs, the long bones recovered at 3 weeks from animals implanted s.c. with human tumors and tissue sections were stained for human PCa cells using anti-human HLA antibodies, HSCs (CD150+CD41-CD48- Lin $^{-}$), and osteoblastic niche cells expressing the osteoblast-specific transcription factor Runx2. Despite the rarity of both disseminated populations, both $\mathrm{PCa}$ cells and HSCs colocalized with Runx2-expressing cells (Figure 3, B and C). Similarly, disseminated PCa cells and HSCs localized close to one another (Figure 3D).

To further validate that the disseminated PCa cells and HSCs colocalize to the same endosteal niche, multiphoton imaging was used to track PCa cell and HSC homing to the BM after transplantation. After transplantation, both cell types colocalized within 5 cell distances of each other, whereas NMPE control cells did not colocalize with HSCs (Figure 4, A-C).

In vitro studies were performed to further characterize the molecular mechanisms used by PCa cells to localize to the HSC niche. HSCs were isolated using the SLAM family of receptors $\left(\mathrm{Lin}^{-} \mathrm{CD} 150^{+} \mathrm{CD} 48^{-} \mathrm{CD} 41^{-} \mathrm{Sca}-1^{+} \mathrm{C}-\mathrm{Kit}^{+}\right.$, referred to herein as SLAM HSCs; refs. 11, 23). In short-term adhesion assays, PCa cells were able to colocalize to a single osteoblast (Figure 4B and Supplemental Figure 4, A and B), which suggests that HSCs and PCa cells may localize extremely close to one another in the BM (but not NMPE control cells; Figure 4F). We have previously noted that Anxa2 expression by osteoblasts plays a central role in niche selection of both HSCs and PCa cells (Supplemental Methods and refs. 13, 24). Here, we noted that both HSCs and PCa cells bound significantly better to Anxa2-expressing osteoblasts (i.e., from $A n x a 2^{+/+}$animals) than to those isolated from Anxa2 $2^{-/-}$animals (Figure 4, D and E). However, NMPE control cells did not bind to either $A n x a 2^{+/+}$or
Anxa2 $2^{-/-}$osteoblasts (Figure 4F). Together, these findings, along with the competitive engraftment data, demonstrated that $\mathrm{PCa}$ cells and HSCs compete for the HSC niche in BM.

Alterations of the endosteal HSC niche regulate PCa metastasis. Changes in osteoblast numbers have been demonstrated to alter the number of HSC niches $(7,15)$. To determine whether changing the number of osteoblastic niche cells influences metastasis, mice were pretreated with PTH $(50-80 \mu \mathrm{g} / \mathrm{kg}$ for 3 weeks $)$, which has been shown to increase endosteal osteoblastic niches and subsequently HSC numbers in BM (Figure 4G; Supplemental Figure 4, C and D; and ref. 7). To ensure no direct effect of PTH on the tumor cells themselves, the animals were rested prior to establishing s.c. primary tumors. After 3 weeks, the number of PCa cells disseminated from the primary tumors into the BM was determined. The data demonstrated that increasing the number of osteoblastic lineage cells also increased the number of metastatic PCa cells in the BM (Figure 4G and Supplemental Figure 4E).

If increasing the number of HSC niches in BM leads to increased number of metastatic PCa cells in BM, then it would be predicted that decreasing the number of niches in BM would decrease the number of metastatic cells in BM. To test the possibility that decreasing the number of osteoblasts (e.g., by decreasing niche size) reduces metastasis, s.c. metastasis assays were performed in which vertebral bodies (vossicles) derived from wild-type mice or mice expressing the Col2.3 $\Delta$-TK transgene (Figure $4 \mathrm{H}$ ) served as the metastatic target. Activation of the osteoblast-specific Col2.3 $\Delta$-TK transgene results in loss of osteoblasts in the presence of ganciclovir (Supplemental Methods and ref. 15). There were no changes in PCa cells derived from the primary tumor in wild-type or Col2.3 $\Delta$-TK vossicles without osteoblastic niche cell ablation (stimulated with 3-8 mg/kg ganciclovir for 3 weeks; Figure $4 \mathrm{H}$ and Supplemental Figure 4F). There were also no changes in disseminated PCa cells in wild-type vossicles treated with ganciclovir or vehicle alone (Figure 4H and Supplemental Figure 4F). Importantly, fewer disseminated metastatic PCa cells were recovered from the Col2.3ム-TK vossicles in animals treated with ganciclovir (Figure $4 \mathrm{H}$ and Supplemental Figure $4 \mathrm{~F}$ ). While the possibility remains that ganciclovir treatment of Col2.3 $\Delta$-TK vossicles affects other cells of the BM microenvironment, these data suggest that metastasis depends on the number of osteoblastic niche in bone.

To determine whether tumor growth in BM is dependent on the osteoblastic HSC niches, tumor cells were directly injected into vossicles prior to implantation (13). Without ganciclovir, the tumors grew at similar rates in wild-type and Col2.3 $\Delta$-TK vossicles. In contrast, tumors did not grow in ganciclovir-treated Col2.3 $\Delta$-TK vossicles, while having no negative effects on primary tumor growth (Figure 5, A and B, and Supplemental Figure 5). Ganciclovir treatment ablated osteoblastic lineage cells in Col2.3 $\Delta$-TK tissues, but had no effect on the number of osteoblastic lineage cells in vehicle-treated Col2.3 $\Delta$-TK or wild-type vossicles (Figure 5, C and D). Importantly, ganciclovir alone had no effect on PCa cell growth in wild-type vossicles, but decreased PCa cell growth in Col2.3 $\Delta$-TK vossicles by inducing apoptosis (Figure $5, \mathrm{E}$ and $\mathrm{F}$ ), which suggests that osteoblastic niche cells are critical for PCa cell growth in bone. Together, these data suggest that PCa metastasis and tumor growth in bone is dependent upon the endosteal osteoblastic niche.

Removing HSCs from the niche increases metastasis. In steady-state conditions, HSCs and hematopoietic progenitor cell (HPCs) circulate in the blood at low frequency, but increase in response to myelosuppressive chemotherapy or growth factors (e.g., G-CSF; 

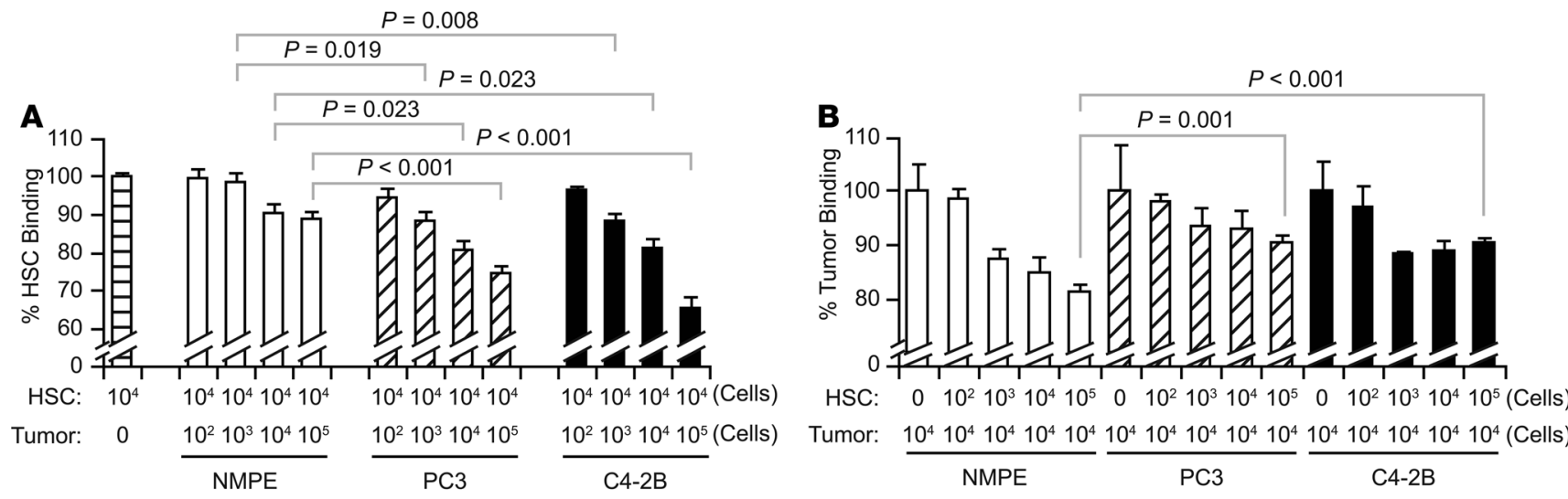

Tumor: $10^{4} 10^{4} 10^{4} 10^{4} 10^{4} 10^{4} 10^{4} 10^{4} 10^{4} 10^{4} 10^{4} 10^{4} 10^{4} 10^{4} 10^{4}$ (Cells)
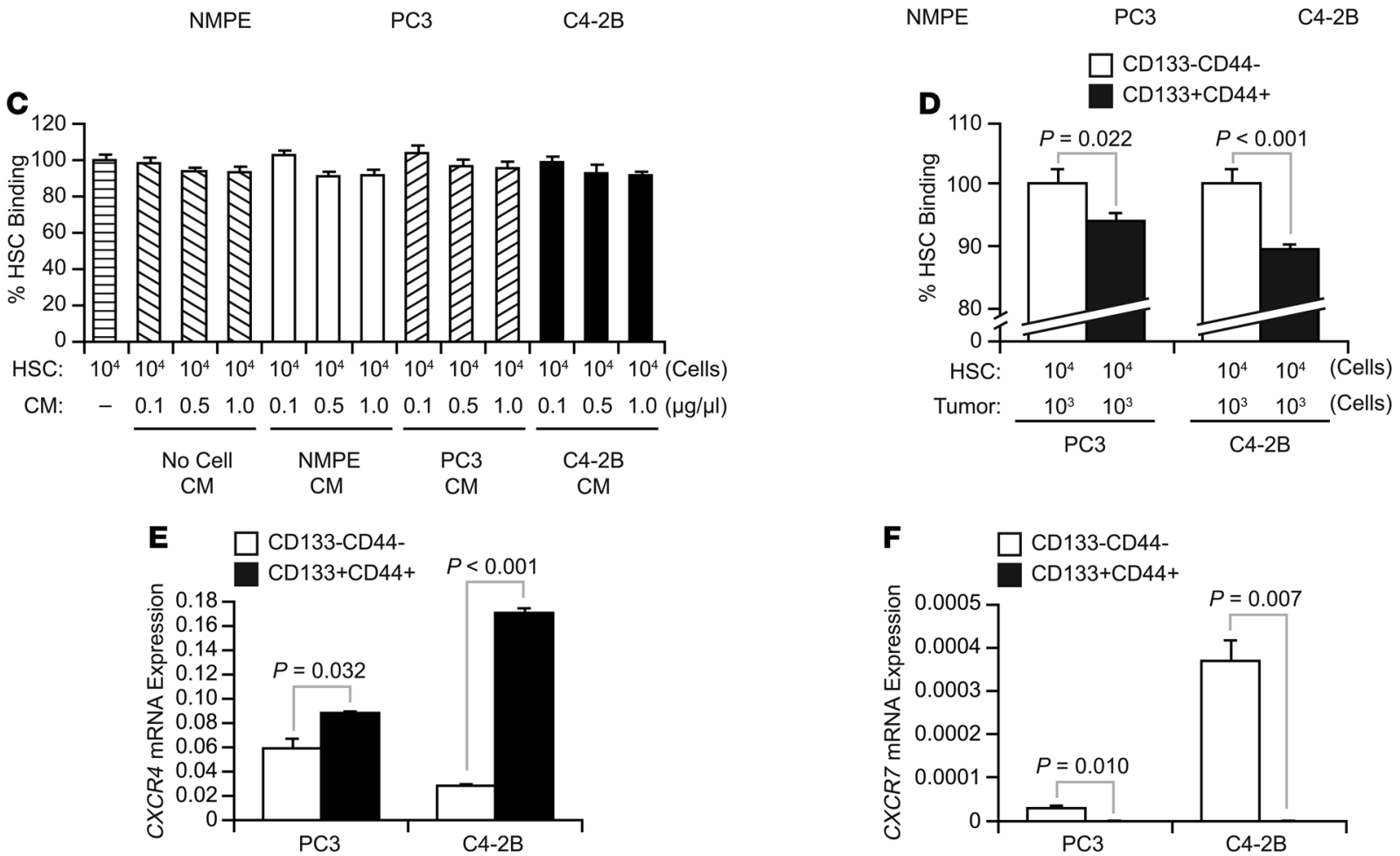

G

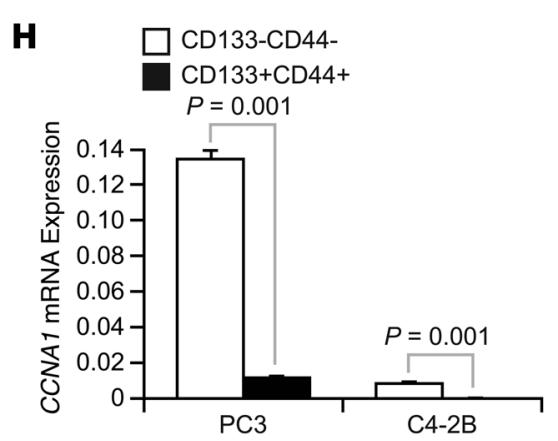

Figure 7

Mechanisms regulating niche competition between PCa cells and HSCs: competition for binding to osteoblasts. (A) Competition binding assays to murine osteoblasts between $10^{4}$ LSK HSCs and 0-105 PCa cells or NMPE control cells. (B) A fixed number of labeled NMPE control cells and PCa cells $\left(10^{4}\right.$ cells) and $0-10^{5} \mathrm{HSCs}$ were layered onto murine osteoblasts. The binding ability of NMPE control cells and PCa cells to osteoblasts in the presence of HSCs was evaluated using a fluorescent plate reader. (C) A fixed number of fluorescently labeled $\mathrm{HSCs}$ (104 cells) was layered onto murine osteoblasts. At the same time, cultures were treated with $0-1 \mu \mathrm{g} / \mu \mathrm{l}$ of medium alone or conditioned medium (CM) derived from NMPE control cells and PCa cells. The binding ability of HSCs was measured by fluorescent plate reader. (D) Competition binding assays between $10^{4} \mathrm{HSCs}$ and $10^{3} \mathrm{CD} 133^{+} \mathrm{CD} 44^{+}$or CD133-CD44- PCa cells. Data are from 3 independent experiments. (E-H) mRNA levels of (E) CXCR4, (F) CXCR7, (G) CCND1, and (H) CCNA1 in CD133+CD44+ or CD133-CD44- PCa cells. Significance of differences was determined by Student's $t$ test (A-D) or Kruskal-Wallis test (E-H). 
Table 1

Frequency of CD133+CD44+ and CD133-CD44- PCa cells

\begin{tabular}{lrrrr} 
Source & \multicolumn{2}{c}{ PC3 } & \multicolumn{2}{c}{ C4-2B } \\
& CD133+CD44+ $^{+}$ & CD133-CD44- $^{-}$ & CD133+CD44+ $^{+}$ & CD133-CD44- \\
In vitro & $0.86 \pm 0.52$ & $38.23 \pm 0.64$ & $1.47 \pm 0.74$ & $24.24 \pm 2.75$ \\
In vivo BM recovered & $29.14 \pm 3.59$ & $6.17 \pm 1.37$ & $37.3 \pm 4.48$ & $7.72 \pm 1.72$ \\
\hline
\end{tabular}

PCa cells recovered from culture were evaluated for CD133 and CD44 expression. Thereafter, $1 \times 10^{6} \mathrm{PCa}$ cells were injected i.c. into SCID mice to establish disseminated cells in the BM. After 24 hours, BM was recovered from the long bones, and hematopoietic lineage cells were depleted using magnetic beads. CD133 and CD44 frequency in disseminated PCa cells (shown as percentages) was evaluated with FACS by gating on HLA-ABC (>99\% PCa positive).

MMP9) that degrade CXCL12 (25). To determine whether loss of CXCL12 could be responsible for the mobilization of PCa cells into the peripheral blood once in the HSC niche, levels of MMP2, MMP9, and CXCL12 were examined after G-CSF treatment. As expected, $\mathrm{G}-\mathrm{CSF}$ induced increases in the expression of MMP2 and MMP9 in the BM of G-CSF-treated tumor-bearing mice and resulted in a substantial decrease in the levels of CXCL12 (Figure 6, $\mathrm{J}$ and $\mathrm{K}$ ). A second major pathway believed to regulate HSC mobilization is through the induction of osteoclastic bone resorption (26). Therefore, we next explored the role of osteo-

ref. 25) and when CXCR4/CXCL12 signaling is interrupted (18). If PCa cells and HSCs compete for the niche, then it should be possible to increase the number of metastatic cells in the niche by vacating HSCs from the niche. To explore this possibility, mechanistic studies were designed to explore whether disseminated PCa cells use the CXCR4/CXCL12 pathway to gain entrance and egress of the HSC niche (18). Here, experimental animals were pretreated for 5 days with $5 \mathrm{mg} / \mathrm{kg}$ AMD3100, an antagonist of CXCR4 that mobilizes HSCs into the peripheral blood to "open” the HSC niche (18). Subsequently, PCa cells were inoculated into the animals by intracardiac (i.c.) injection to establish disseminated tumor cells (Figure 6A). More PCa cells had homed to the BM 24 hours later in the bones of the AMD3100-pretreated animals than in the vehicletreated animals (Figure 6B and Supplemental Figure 6A). These data suggest that HSC occupancy of the niche limits metastasis.

$P C a$ cells can be mobilized out of the HSC niche and into the blood using HSC mobilizing agents. If PCa cells target the HSC niche, then it should also be possible to induce PCa cells to reenter the peripheral circulation by interfering with CXCR4/CXCL12 signaling, as has been shown for HSCs. To explore this possibility, disseminated PCa cells were first established in bone after tumor implantation (Figure 6C). After removal of the primary tumors, the animals were rested and then treated with AMD3100 or vehicle. Blood was collected 24 hours after the last AMD3100 injection, and the number of circulating PCa cells determined (Figure 6C). More circulating PCa cells were found in blood after treatment with AMD3100 versus vehicle (Figure 6D and Supplemental Figure 6B). AMD3100 mobilized the HSCs from the BM by inhibiting mRNA expression of both CXCR4 and CXCR7 in the HSCs (Figure 6, E-G). These data suggest that disseminated PCa cells target the HSC niche through the CXCR4/CXCL12 pathway.

G-CSF is another agent that is used clinically to mobilize HSCs out of the niche and into the peripheral blood to improve stem cell collection prior to BMT (25). Here, the mobilization studies were repeated using recombinant G-CSF. G-CSF mobilized PCa cells from the HSC niche and into the peripheral blood with higher frequency than in vehicle-treated control animals (Figure 6H and Supplemental Figure 6C). To exclude a direct effect of G-CSF on PCa cells, we also confirmed that PCa cells did not express G-CSF receptors, nor did G-CSF induce PCa cell proliferation (Supplemental Figure 6, D-G). As expected, G-CSF mobilized the HSCs from the BM (Figure 6I), which suggests that PCa cells use the same mechanisms as HSCs and HPCs to enter the peripheral circulation.

Mobilization of HSCs and HPCs by G-CSF is thought to occur through the induction of 2 pathways. The first is through the production of enzymes (e.g., CD26, cathepsin G, elastase, MMP2, and clasts in mobilization of PCa cells from the niche. Interestingly, G-CSF induced osteoclastogenesis in the presence of disseminated PCa cells (Supplemental Figure 6, H and I). Conversely, AMD3100 which does not mobilize HSCs by activating osteoclastogenesis, but rather by interfering directly with CXCR4/CXCL12 binding did not active osteoclasts to mobilize PCa cells, as predicted (Supplemental Figure 6, J and K; and ref. 27). Together, these data suggest that disseminated PCa cells compete with HSCs for the niche using the same molecular mechanisms as do HSCs to gain access and egress of the niche (e.g., CXCR4/CXCL12).

Direct cell-to-cell competition for niche binding favors PCa cells. A second molecular mechanism for the competition between HSCs and PCa cells for the niche may be that disseminated PCa cells can displace or outcompete HSCs for adhesion of niche-binding elements. Accordingly, we performed competitive binding assays between HSCs and PCa cells. PCa cells blocked HSC binding to osteoblasts, whereas NMPE control cells were less able to prevent HSC binding to osteoblasts (Figure 7A). Recently, we demonstrated that Anxa2 expressed by osteoblasts is a critical molecule used by both HSCs and PCa cells for binding to osteoblasts $(13,24)$. The competitive binding assays were therefore repeated using Anxa2 as the binding target. As expected, PCa cells blocked the binding of HSCs to Anxa2 better than did the NMPE control cells (Supplemental Figure 7A). Conversely, HSCs more efficiently prevented the binding of NMPE cells to osteoblasts and Anxa2 than they blocked PCa cell binding (Figure 7B and Supplemental Figure 7B). These effects required direct cell-to-cell interactions, as soluble factors present in the conditioned medium of PCa cells alone were unable to alter HSC binding to osteoblasts (Figure 7C).

When PCa cells expressing a putative PCa stem cell phenotype $\left(\mathrm{CD} 133^{+} \mathrm{CD} 44^{+}\right)$were isolated from culture and used in competitive adhesion assays with HSCs, they were better able to block HSC binding to osteoblasts compared with CD133-CD44- cells (Figure 7D). These $\mathrm{CD}_{133}{ }^{+} \mathrm{CD} 44^{+}$cells expressed higher levels of CXCR4, but lower CXCR7 (Figure 7, E and F), and progressed through the cell cycle slower than did PCa cells expressing a CD133-CD44- phenotype, based on expression of CCNA1 and CCND1 (encoding cyclin A1 and cyclin D1, respectively; Figure 7, G and H). Intriguingly, although the $\mathrm{CD} 133^{+} \mathrm{CD} 44^{+}$cells represented a very small fraction of the total cells found in culture $(0.86 \% \pm 0.52 \%$ of PC 3 and $1.47 \% \pm 0.74 \%$ of $\mathrm{C} 4-2 \mathrm{~B}$ in vitro), the frequency of this population was significantly enriched in BM 24 hours after i.c. injection (Table 1).

PCa cell occupation of the HSC niche alters the phenotype of HSCs and displaces HSCs from the niche. A third mechanism that may assist $\mathrm{PCa}$ cells in the competition for the HSC niche may be that tumor cells themselves have direct and indirect effects on HSCs. PCa cells may 


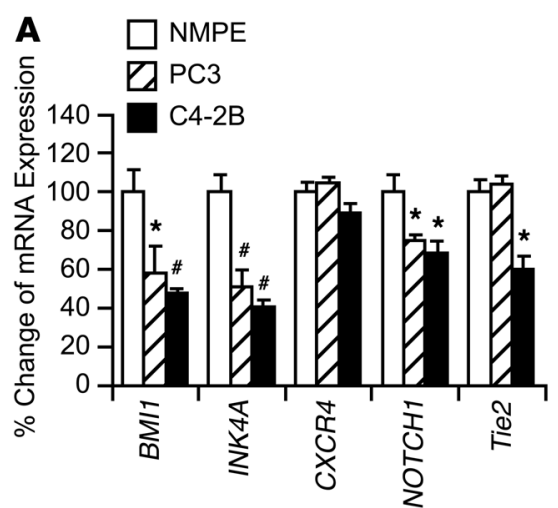

$\mathbf{B}$
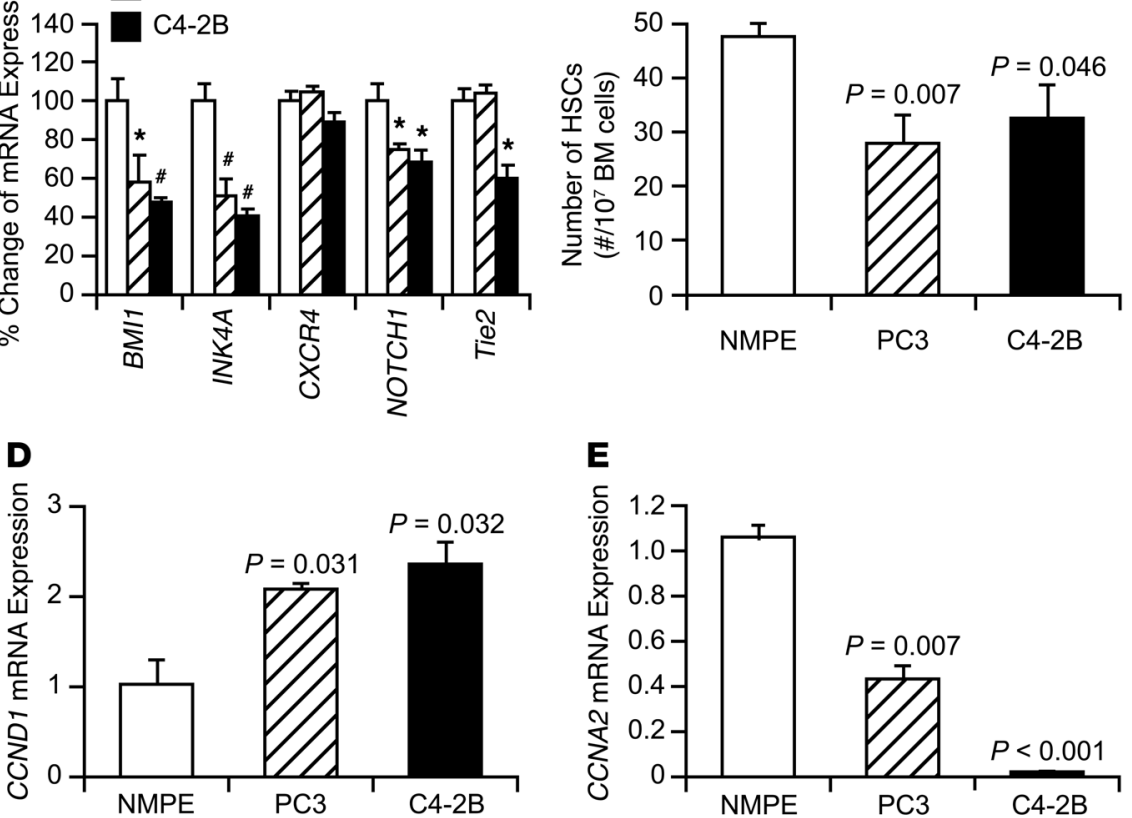

G

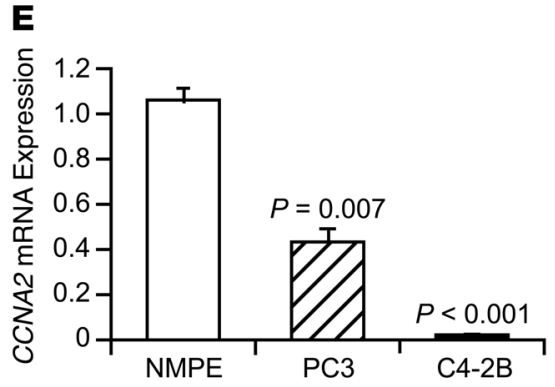

C

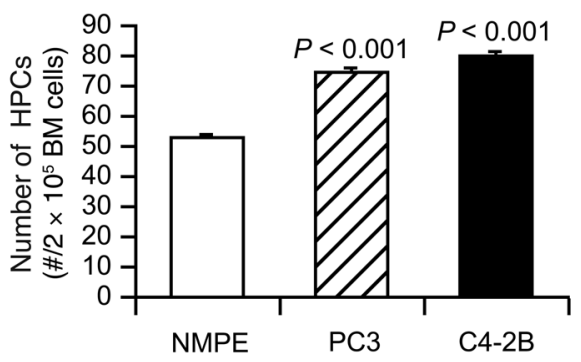

F

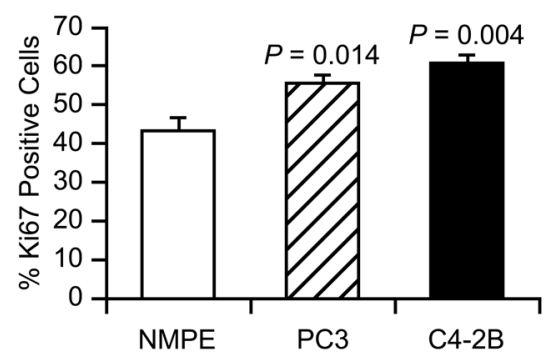

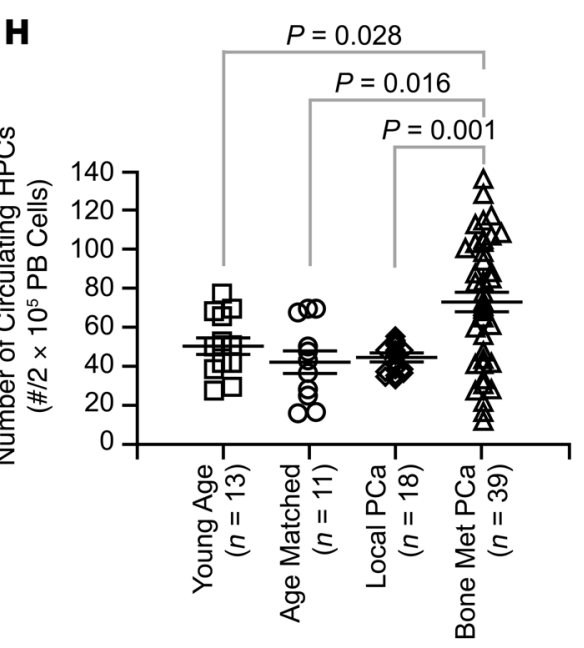

Figure 8

Mechanisms regulating niche competition between PCa cells and HSCs: PCa cells drive HSCs out from the HSC niche. (A-G) SCID mice were implanted either NMPE cells or PCa cells (PC3 or C4-2B) ( $n=5$ per group). After 3 weeks, the BM cells were collected. (A) Expression of stem cell survival and cell-to-cell adhesion genes in SLAM HSCs was evaluated by quantitative real-time RT-PCR $\left(n=5\right.$ per group). ${ }^{*} P<0.05$, $\# P<0.01$ versus NMPE. (B) SLAM HSC numbers in BM were counted by FACS, and (C) HPC numbers were determined using colony-forming assays. (D and E) mRNA levels of (D) CCND1 and (E) CCNA2 in HSCs. (F) Cell cycle (Ki-67-positive cells) and (G) apoptotic state (PE-conjugated annexin V/7-ADD) of HPCs were analyzed by gating on Lin-Sca-1+ populations. (H) Peripheral blood was collected from subjects with local PCa disease $(n=18,61.6 \pm 9.2$ years of age) and disseminated PCa disease ( $n=39,68.1 \pm 11.1$ years of age). The number of hematopoietic colonies was compared with that in healthy controls (young age, $n=13$, 34.3 \pm 5.6 years; age-matched, $n=11,62.4 \pm 7.1$ years). Significance of differences was determined by Kruskal-Wallis test (A, D, and $\mathbf{E})$ or Student's $t$ test $(\mathbf{B}, \mathbf{C}$, and $\mathbf{F}-\mathbf{H})$; $P$ values in B-F are versus NMPE.

be able to drive HSCs to maturity and into HPC populations so that they eventually vacate the niche. To explore this possibility, mice were implanted with metastatic PCa cells or NMPE control cells to establish disseminated tumors in the BM. HSCs isolated from animals with disseminated PCa cells expressed lower levels of the niche adhesion molecules (NOTCH1, ref. 7; TIE2, ref. 9) and transcription factors known to regulate HSC self-renewal and proliferation (BMI1 and INK4A; Figure 8A and refs. 28, 29). These results suggest that disseminated PCa cells reduce HSC numbers by altering HSC self-renewal. Consistent with these observations, fewer HSCs were found in the tumor-bearing animals (Figure 8B). One mechanism to explain this reduction is that HSCs are driven into HPCs pools. Indeed, more HPCs were recovered from the BM of tumor-bearing animals than from that of controls (Figure 8C). As expected, HSCs and HPCs derived from tumor-bearing animals were induced into a cell-cycling state (Figure 8, D-F), while there 


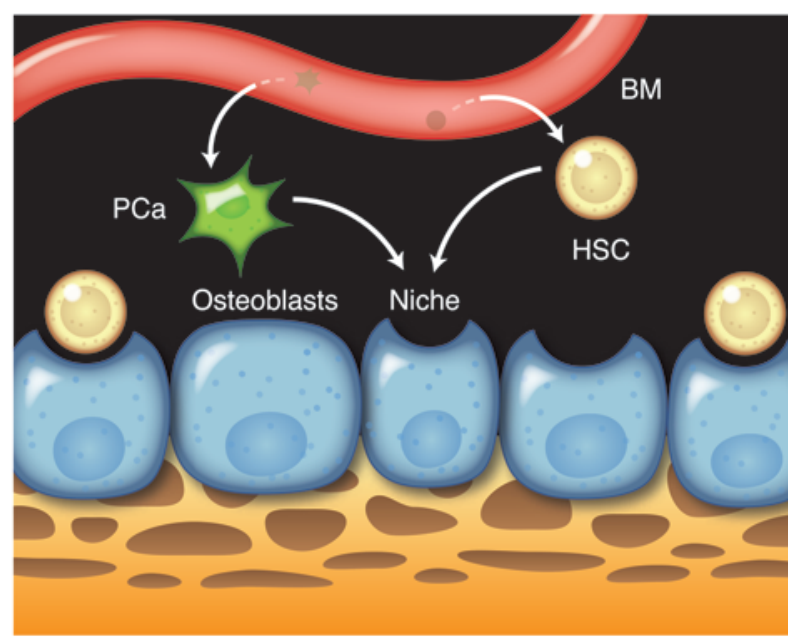

Figure 9

Competition between disseminated PCa cells and HSCs for the endosteal niche facilitates metastasis. The endosteal (or osteoblast) niche is thought to maintain HSCs quiescence and regulate differentiation. It is also appreciated that many solid tumors metastasize to the bone. Our hypothesis is that metastatic cells shed from a primary tumor compete with HSCs to engage the endosteal niche, which suggests that solid tumors use the HSC niche as metastatic niche. Once in the niche, disseminated cells may remain in a quiescent/dormant state for extended periods of time. Eventually, however, metastatic growth exceeds the niche's regulatory capacity, and clinically relevant disease occurs.

was no effect of PCa cells on HPC apoptosis (Figure 8G). Although it is not presently clear what molecules are responsible for these activities, HSCs were chemotactic toward PCa cells themselves, but PCa cells did not have similar effects on HSCs, and HSCs were able to enhance the growth of PCa cells (Supplemental Figure 8, A-C).

If PCa cells compete for the niche with HSCs and HPCs and drive these cells into the circulation, then it would be expected that more HSCs or HPCs would be present in the circulation of men with metastatic bone disease than in age-matched controls. Therefore, HPC assays were performed on peripheral blood collected from subjects with disseminated PCa bone disease. As expected, more circulating HPCs were found in subjects with disseminated disease compared with local disease (defined as PCa with no imaging evidence of metastases and PSA $<15 \mathrm{ng} / \mathrm{ml}$ ) and age-matched controls (Figure $8 \mathrm{H}$ ). Although other explanations are possible (e.g., inflammatory cytokines or cachexia), these data are consistent with the hypothesis that disseminated tumor cells target the HSC niche and displace HPCs into the peripheral blood. Together, these data strongly suggest that PCa cells target the HSC niche during metastasis.

\section{Discussion}

Our data suggest that the HSC niche serves as a specific site where disseminated cells from PCa gain footholds in the BM (Figure 9). These studies showed, for the first time to our knowledge, that disseminated cells from solid tumors are able to compete with HSCs for occupancy of the HSC niche and colocalize with HSCs in the BM. In fact, by mobilizing HSCs out of the niche, or by altering the size and/or number of the endosteal HSC niches, we were able to alter the number of metastatic cells present in the BM. We observed at least 3 molecular mechanisms that may be operative during metastasis and facilitate disseminated tumor cells' ability to gain footholds in the
HSC niche. First, PCa cells use the CXCR4/CXCL12 pathway to gain access and egress from the HSC niche area, as do HSCs themselves. In fact, we previously demonstrated that blocking this pathway, or blocking the alternative CXCL12 receptor CXCR7, prevents metastasis and PCa growth in bone $(12,19,20)$. A second mechanism that may provide PCa cells an advantage over HSCs in establishing themselves in the niche is a superior ability to bind to and/or engage receptors that HSCs use to localize to the niche. Indeed, we recently demonstrated that both HSCs and PCa cells both bind to Anxa2 and that blocking the receptor for Anxa2 prevents homing to the $\operatorname{BM}(13,24)$. Finally, we demonstrated that HSCs themselves were targets of metastatic cells, as they were driven into progenitor pools, into the peripheral blood, or both, and may be able to support the growth of the cancer within the niche. Together, these data suggest that metastatic PCa cells, and perhaps cells from other tumors, serve as molecular parasites of the HSC niche.

Little is known about the postdissemination and niche engagement events activated by PCa cells to coopt the HSC niche. Based on the average length of time for relapse to occur after primary therapy (surgery or radiation; refs. 30,31), it is possible that the HSC niche is able to regulate dormancy of tumor cells for extended periods. In fact, clinically relevant metastases are relatively rare compared with the number of disseminated tumor cells (32). Because the endosteal HSC niche plays a crucial role in maintaining stemness, the ability of tumor cells to engage the endosteal HSC niche may facilitate the ability of tumor cells to establish metastatic foci in bone while remaining dormant over long time periods $(33,34)$. Moreover, it has been appreciated that preosteoblasts are critical compartment of the HSC niche rather than mature osteoblasts $(35,36)$. However, further studies are needed to determine whether preosteoblasts serve as the solid tumor niche.

Similarly, what leads to the loss of tumor dormancy is unknown. One prospect worth considering is that there are substantial niche reserves in the BM for HSCs. However, a recent study demonstrated that HSC replacement of the niche constantly occurs under normal physiological condition (37). Perhaps as disseminated tumor cells proliferate slowly over time, the capacity of the niche is overwhelmed, leading to clinically relevant disease. In fact, peripheral blood changes - including infection, anemia, and thrombocytopenia, indicative of HSC failure - are late-stage events, but are commonly observed at death in individuals with metastatic disease (38). Other possibilities are that the tumor cells themselves become resistant to the dormancy signals provided by the niche over time (38).

In 1889, Paget proposed a "seed and soil" metaphor to explain the marked affinity of cancer cells for different tissues (39). It has previously been reported that VEGFR1-expressing BM cells participate in the process by establishing premetastatic niches (14). Although inflammatory cytokines released by tumors might affect HSCs directly, our data suggest that there may be other biologic targets that tumors use to establish disseminated metastases in bone. Our work has the potential to facilitate better understanding of the molecular events involved in bone metastases and to lead to new therapeutic avenues for an incurable disease.

\section{Methods}

Further information can be found in Supplemental Methods.

Cell culture. The PC3 (CRL-1435) PCa cell line was obtained from the American Type Culture Collection. The metastatic subline LNCaP C4-2B was originally isolated from a lymph node of a patient with disseminated bony and lymph node involvement (40). Normal human NMPE control 
cell lines were obtained from patients undergoing prostatectomy in accordance with the Investigation Review Board of University of Michigan. The tissue was collected from a distal location from the tumor (within the prostate). This cell line is morphologically and pathologically distinct from the tumor. PCa cell lines and NMPE cell lines were cultured in RPMI 1640 (Invitrogen) and DMEM (Invitrogen), respectively. All cultures were supplemented with $10 \%(\mathrm{v} / \mathrm{v})$ FBS (Invitrogen) and $1 \%(\mathrm{v} / \mathrm{v})$ penicillin-streptomycin (Invitrogen) and maintained at $37^{\circ} \mathrm{C}, 5 \% \mathrm{CO}_{2}$, and $100 \%$ humidity.

In vivo $P$ Ca metastasis assays. Male 5- to 7-week-old CB.17 SCID mice were implanted with $2 \times 10^{5}$ cells $(\mathrm{PCa})$ within sterile collagen scaffolds $(3 \times 3$ $\times 3 \mathrm{~mm}^{3}$; Gelfoam; Pharmacia and Upjohn) in the mid-dorsal region of each mouse. In some cases, NMPE cells $\left(2 \times 10^{5}\right.$ cells $)$ were used. When the animals were sacrificed, tissue samples from the animals' left organ/tissue (original scaffold, calvaria, mandible, humeri, femur, tibia, pelvis, spine, and peripheral blood) were dissected and stored at $-80^{\circ} \mathrm{C}$ until genomic DNA extraction. The number of disseminated cells was assessed by QPCR (16). Further normalization was performed for differences in mouse tissue density using murine $\beta$-actin primers.

BMT. NOD/SCID mice (CD45.1) were implanted s.c. with $2 \times 10^{5} \mathrm{PCa}$ cells or control cells. At 3 weeks, s.c. tumors were removed. After 5 days, the NOD/SCID mice were injected with $2 \times 10^{5}$ mononuclear BM donor cells (CD45.2 C57BL/ 6 mice) into the left retro-orbital sinus without irradiation. At 4, 8, 12, and 16 weeks after BMT, the percentage of blood cells bearing the CD45.2 and CD45.1 phenotypes was determined in peripheral blood obtained from the tail veins of individual recipient mice stained together with Mac1 (macrophage), Gr-1 (granulocyte), B220 (B cell), and CD3e ( $\mathrm{T}$ cell) lineage markers. Samples were analyzed by FACS Vantage dual laser flow cytometry (Becton Dickinson).

Survival assays. SCID mice were lethally irradiated with 700 cGy (2×350 cGy) and injected with either $100 \mu \mathrm{l}$ of $0.9 \%$ saline (negative control) or mononuclear BM donor cells $\left(4 \times 10^{5}\right.$ cells $\left./ 100 \mu \mathrm{l}\right)$ into the left retro-orbital sinus. In some cases, animals were injected i.c. with $4 \times 10^{6}$ irradiated (8 Gy; $2 \times 4$ Gy) PC3 or C4-2B PCa cells or with irradiated (8 Gy; $2 \times 4$ Gy) NMPE control cells. Moribund animals were humanely killed as required. Survival observations were maintained for 60 days.

In vivo competitive engraftment assays. NOD/SCID Il2 $\mathrm{rg}^{-1-}$ mice were sublethally irradiated with $300 \mathrm{cGy}(2 \times 150 \mathrm{cGy})$ and injected with either $100 \mu \mathrm{l}$ of $0.9 \%$ saline (negative control) or human $\mathrm{BM} \mathrm{CD} 34^{+}$cells $\left(2 \times 10^{5}\right.$ cells $\left./ 100 \mu \mathrm{l}\right)$ into the left retro-orbital sinus. In some cases, animals received $2 \times 10^{6}$ irradiated ( $8 \mathrm{~Gy} ; 2 \times 4 \mathrm{~Gy}$ ) PC 3 or C4-2B PCa cells or irradiated ( 8 Gy; $2 \times 4$ Gy) NMPE control cells, injected i.c. to avoid their being trapped in the lungs. At $4,8,12$, and 16 weeks after transplantation, the percentage of human $\mathrm{CD} 34^{+}$cell engraftment was determined in peripheral blood obtained from the tail veins of individual recipient mice stained with human CD45. Samples were analyzed by FACS Vantage dual laser flow cytometry (Becton Dickinson).

Isolation of HSCs. HSCs were isolated as previously described (23). The BM cells were flushed from the femurs and tibias. Cells were incubated first with a biotinylated anti-Lin (CD5, CD45R [B220], CD11b, Gr-1 [Ly- 6G/C], and Ter-119) antibody cocktail (Miltenyi Biotec) for 10 minutes at $4^{\circ} \mathrm{C}$, then rinsed and stained with an antibody cocktail of allophycocyanin-conjugated anti-Sca-1 (clone D7; eBioscience), PE/Cy7-conjugated anti-c-Kit (clone 2B8; BioLegend), PE-conjugated anti-CD150 (clone TC15-12F12.2; BioLegend), FITC-conjugated anti-CD41 (clone MWReg30; BD Biosciences), FITC-conjugated anti-CD48 (clone BCM-1; BD Biosciences) and FITCconjugated anti-biotin antibodies (Miltenyi Biotec) for another 20 minutes at $4^{\circ} \mathrm{C}$. HSCs were sorted on a FACS Vantage dual laser flow cytometer by gating on SLAM HSCs (i.e., $\mathrm{Lin}^{-} \mathrm{CD} 150^{+} \mathrm{CD} 41^{-} \mathrm{CD} 48^{-} \mathrm{Sca}-1^{+} \mathrm{c}-\mathrm{Kit}^{+}$). In some cases, murine LSK HSCs were obtained using first a Lineage Cell Depletion Kit magnetic labeling system with biotinylated (CD5, CD45R [B220],
CD11b, Gr-1 [Ly-6G/C], and Ter-119) and anti-biotin MicroBeads (Miltenyi Biotec). Positive immunoselection was performed with PE-conjugated anti-Sca-1 and FITC-conjugated anti-c-Kit (BD Biosciences - Pharmingen) and sorted on a FACS Vantage dual laser flow cytometer.

Murine osteoblasts. Calvariae of mice (1-4 days old) were dissected, isolated from periosteum, and subjected to sequential digestions of 20,40, and 90 minutes in collagenase A ( $2 \mathrm{mg} / \mathrm{ml}$; Roche Molecular Biochemicals) with $0.25 \%$ trypsin (Invitrogen), as previously described $(13,41)$. Cells from the third digestion were plated in $\alpha$-MEM (Invitrogen) with 10\% (v/v) FBS and $1 \%(\mathrm{v} / \mathrm{v})$ penicillin and streptomycin.

Vossicle transplant. Lumbar vertebrae were isolated from 4- to 7-day-old mice. The vertebrae were sectioned into single vertebral bodies (i.e., vossicles). SCID mice were used as transplant recipients. 2 vossicles per mouse were implanted into s.c. pouches as previously described $(13,16)$.

In vitro colocalization assays. Murine osteoblasts labeled with CellTracker Blue CMAC (Invitrogen) were cultured in Lab-Tek II 4-chamber slides (Nalge Nunc International) at $1 \times 10^{4}$ cells/chamber. After 24 hours, SLAM HSCs labeled with CellTracker Red CMTPX (Invitrogen) and PC3 and C4-2B PCa cells labeled with CellTracker Green CMFDA (Invitrogen) were added to this culture system (200 cells/chamber). Thereafter, the cultures were incubated in an atmosphere of $5 \% \mathrm{CO}_{2}$ and $95 \% \mathrm{O}_{2}$ at $37^{\circ} \mathrm{C}$ for 2 hours. Fluorescence was observed under a FV500 confocal laser-scanning microscope (Olympus).

In vivo colocalization assays. Vossicles were implanted into SCID mice. After 3 weeks, a total of $1 \times 10^{6} \mathrm{PC} 3$ cells expressing green fluorescent protein were injected i.c. After 24 hours, animals received i.v. injection of LSK HSCs $\left(1 \times 10^{6}\right.$ cells $)$ into the left retro-orbital sinus labeled with Qtracker 655-nm nontargeted quantum dots (Quantum Dot Corp.). Vossicles were subsequently harvested and imaged by multiphoton microscopy or confocal microscopy, as previously described (16).

PTH treatment. Animals were administered human PTH $\left(\mathrm{hPTH}_{1-34}\right.$; Bachem) or vehicle ( $0.9 \%$ saline) by i.p. injection at $50-80 \mu \mathrm{g} / \mathrm{kg}(100 \mu \mathrm{l})$ per day for 3 weeks (41).

Ganciclovir treatment. Vossicle-implanted animals were administered ganciclovir (Cytovene-IV; Roche) or vehicle ( $0.9 \%$ saline) by i.p. injection at $3-8 \mathrm{mg} / \mathrm{kg}(100 \mu \mathrm{l})$ per day for 3 weeks.

AMD3100 treatment. AMD3100 (Sigma-Aldrich) or vehicle (0.9\% saline) was administered by i.p. injection at $5 \mathrm{mg} / \mathrm{kg}(100 \mu \mathrm{l})$ per day for 5 days. In some cases, animals were injected with $1 \times 10^{6} \mathrm{PC} 3$ and C4-2B PCa cells i.c. 24 hours after the last AMD3100 injections. After 24 hours, animals were sacrificed, and PCa cells in the BM were assessed by QPCR.

G-CSF treatment. Animals were administered G-CSF (Neupogen; Amgen) or vehicle $(0.9 \%$ saline $)$ i.p. at $250-300 \mu \mathrm{g} / \mathrm{kg}(100 \mu \mathrm{l})$ per day for 5 days.

Immunohistochemistry. Vossicles and murine long bones were stained with anti-cytokeratin antibody (diluted 1:250, rabbit polyclonal; Abcam) and hematoxylin and eosin as previously described (41).

Bioluminescent imaging. Bioluminescent imaging was performed as previously described through the University of Michigan Small Animal Imaging Resource facility (13).

Methylcellulose cultures. BM cells were collected from control or experimentally treated SCID mice $(n=5)$. Peripheral blood was collected from subjects with disseminated bone PCa disease, subjects with local PCa disease, and healthy volunteers. Peripheral blood mononuclear cells were isolated from whole blood by density gradient centrifugation using Ficoll-Paque PLUS (StemCell Technologies). Approval was obtained from the University of Michigan's Investigation Review Board, and written informed consent was obtained from participating subjects.

BM or blood cells $\left(2 \times 10^{5}\right.$ cells $)$ were plated onto $35-\mathrm{mm}$ tissue culture dishes in methylcellulose with recombinant cytokines for colony assays of murine cells (Methocult GF M3434; StemCell Technologies) or human 
cells (Methocult GF H4434; StemCell Technologies). The total number of colonies was counted at day 14 after plating.

Transwell chemotaxis assays. Cell invasion assays were performed in dualchambered Transwell plates (Costar Corp). PC3 and C4-2B PCa cells or NMPE control cells $\left(2.5 \times 10^{5}\right.$ cells $)$ were seeded onto the top well of an $8-\mu \mathrm{m}$ dual chamber, and LSK HSCs $\left(2.5 \times 10^{5}\right.$ cells $)$ were placed in the bottom well of the chamber as chemotaxis targets. In some cases, LSK HSCs $\left(2.5 \times 10^{5}\right.$ cells) were seeded onto the top well of $5-\mu \mathrm{m}$ dual chambers, and PC 3 or $\mathrm{C} 4-2 \mathrm{~B}$ PCa cells or NMPE control cells $\left(2.5 \times 10^{5}\right.$ cells $)$ were placed in the bottom well. Cells seeded on the top well were labeled with $2.5 \mathrm{mg} / \mathrm{ml}$ of the lipophilic dye carboxyfluorescein diacetate (Invitrogen) prior to assays. Spontaneous invasion was compared with invasion supported by cells at bottom wells. The plates were incubated at $37^{\circ} \mathrm{C}$ in $95 \%$ humidity and $5 \% \mathrm{CO}_{2}$. At the termination of the assay (4 hours), chambers were removed, and fluorescence was quantified by fluorescent plate reader (Molecular Devices).

Proliferation assays. Proliferation assays were performed as previously described (13). Luciferase-labeled PCa cells (5,000 cells/well) were plated onto 96-well plates in growth medium with $0.1 \%$ FBS. The next day, LSK HSCs ( $0-5,000$ cells/well) were added to the wells. Thereafter, cultures were incubated for 3 days. Proliferation was determined using a CCD IVIS system with a 50-mm lens (Xenogen Corp.), and the results were analyzed using LivingImage software (Xenogen Corp.).

In vitro binding assays. Cell-to-cell binding assays were performed as previously described (24). Murine calvarial osteoblasts were plated onto 96-well plates at a concentration of $1 \times 10^{4}$ cells/well $(100 \mu \mathrm{l} /$ well $)$ in growth medium, and the cultures were incubated for 2 days. A fixed number of LSK HSCs ( $10^{4}$ cells) labeled with fluorescent dyes (CFDA; Invitrogen) and increasing numbers of PCa cells $\left(0-10^{5}\right.$ cells) or NMPE control cells $\left(0-10^{5}\right.$ cells) were layered onto murine osteoblasts. Binding assays were performed in PBS containing $\mathrm{Ca}^{2+} / \mathrm{Mg}^{2+}$, where the cells were added to a final reaction volume of $100 \mu \mathrm{l}$ at $4{ }^{\circ} \mathrm{C}$. After washing, remaining fluorescence was quantified as a measure of HSC binding by fluorescent plate reader (Molecular Devices). In some cases, PCa cells were first sorted into $\mathrm{CD} 133^{+} \mathrm{CD} 44^{+}$and $\mathrm{CD} 133^{-} \mathrm{CD} 44^{-}$fractions, and binding assays were performed.
Statistics. All numerical data are expressed as mean \pm SEM. Statistical analysis was performed by ANOVA or unpaired 2-tailed Student's $t$ test using GraphPad Instat (GraphPad). For QPCR assays, Kruskal-Wallis test and Dunn multiple-comparisons test was used. For survival assays, KaplanMeier survival analysis was used, and log-rank test was performed using GraphPad Prism (GraphPad) to determine differences between survival curves. For all analyses, a $P$ value less than 0.05 was considered significant.

\section{Acknowledgments}

We thank Laurie K. McCauley and Evan T. Keller for scientific discussions. We also thank Zhuo Wang, Chris Neeley, Taocong Jin, Rhonda L. Hotchkin, and Matt Craig (University of Michigan, Ann Arbor, Michigan, USA) for technical and logistical support; David W. Rowe (University of Connecticut Health Center, Farmington, Connecticut, USA) for Col2.3 $\Delta$-TK mice; Katherine A. Hajjar (Weill Medical College of Cornell University, New York, New York, USA) for Anxa2-/mice; and Chris Jung (University of Michigan, Ann Arbor, Michigan, USA) for artwork. This work was directly supported by a Pediatric Oncology Research Fellowship (to Y. Shiozawa), the Charles Eliot Ware Memorial Fellowship (to A.M. Havens), the National Cancer Institute (grant CA093900, to K.J. Pienta and R.S. Taichman), the Department of Defense (to K.J. Pienta and R.S. Taichman), and the Prostate Cancer Foundation (to K.J. Pienta and R.S. Taichman). K.J. Pienta receives support as an American Cancer Society Clinical Research Professor, NIH SPORE in prostate cancer grant P50 CA69568, and Cancer Center support grant P30 CA46592.

Received for publication April 20, 2010, and accepted in revised form January 12, 2011.

Address correspondence to: Russell S. Taichman, Department of Periodontics and Oral Medicine, University of Michigan School of Dentistry, Room 3307, 1011 North University Avenue, Ann Arbor, Michigan 48109-1078, USA. Phone: 734.764.9952; Fax: 734.763.5503; E-mail: rtaich@umich.edu.
1. Roodman GD. Mechanisms of bone metastasis NEngl J Med. 2004;350(16):1655-1664.

2. Muller A, et al. Involvement of chemokine receptors in breast cancer metastasis. Nature. 2001; 410(6824):50-56.

3. Taichman RS, Cooper C, Keller ET, Pienta KJ, Taichman NS, McCauley LK. Use of the stromal cell-derived factor-1/CXCR4 pathway in prostate cancer metastasis to bone. Cancer Res. 2002;62(6):1832-1837.

4. Wilson A, Trumpp A. Bone-marrow haematopoieticstem-cell niches. Nat Rev Immunol. 2006;6(2):93-106.

5. Yin $\mathrm{T}, \mathrm{Li} \mathrm{L}$. The stem cell niches in bone. J Clin Invest. 2006;116(5):1195-1201.

6. Taichman RS, Emerson SG. Human osteoblasts support hematopoiesis through the production of granulocyte colony-stimulating factor. J Exp Med. 1994;179(5):1677-1682.

7. Calvi LM, et al. Osteoblastic cells regulate the haematopoietic stem cell niche. Nature. 2003; 425(6960):841-846.

8. Zhang J, et al. Identification of the haematopoietic stem cell niche and control of the niche size. Nature. 2003;425(6960):836-841.

9. Arai $\mathrm{F}$, et al. Tie2/angiopoietin-1 signaling regulates hematopoietic stem cell quiescence in the bone marrow niche. Cell. 2004;118(2):149-161.

10. Taichman RS. Blood and bone: two tissues whose fates are intertwined to create the hematopoietic stem-cell niche. Blood. 2005;105(7):2631-2639.

11. Kiel MJ, Yilmaz OH, Iwashita T, Terhorst C, Morrison SJ. SLAM family receptors distinguish hematopoietic stem and progenitor cells and reveal endothelial niches for stem cells. Cell. 2005; 121(7):1109-1121.

12. Wang J, et al. The role of CXCR7/RDC1 as a chemokine receptor for CXCL12/SDF-1 in prostate cancer. J Biol Chem. 2008;283(7):4283-4294.

13. Shiozawa Y, et al. Annexin II/annexin II receptor axis regulates adhesion, migration, homing, and growth of prostate cancer. J Cell Biochem. 2008; 105(2):370-380.

14. Kaplan RN, et al. VEGFR1-positive haematopoietic bone marrow progenitors initiate the pre-metastatic niche. Nature. 2005;438(7069):820-827.

15. Visnjic D, Kalajzic Z, Rowe DW, Katavic V, Lorenzo J, Aguila HL. Hematopoiesis is severely altered in mice with an induced osteoblast deficiency. Blood. 2004;103(9):3258-3264.

16. Havens AM, et al. An in vivo mouse model for human prostate cancer metastasis. Neoplasia. 2008; 10(4):371-380.

17. Papayannopoulou T. Current mechanistic scenarios in hematopoietic stem/progenitor cell mobilization. Blood. 2004;103(5):1580-1585.

18. Broxmeyer HE, et al. Rapid mobilization of murine and human hematopoietic stem and progenitor cells with AMD3100, a CXCR4 antagonist. J Exp Med. 2005;201(8):1307-1318.

19. Sun YX, et al. Expression of CXCR4 and CXCL12 (SDF-1) in human prostate cancers (PCa) in vivo. J Cell Biochem. 2003;89(3):462-473.

20. Sun YX, et al. Skeletal localization and neutraliza- tion of the SDF-1(CXCL12)/CXCR4 axis blocks prostate cancer metastasis and growth in osseous sites in vivo. J Bone Miner Res. 2005;20(2):318-329.

21. Sun YX, Fang M, Wang J, Cooper CR, Pienta KJ, Taichman RS. Expression and activation of alpha $\mathrm{v}$ beta 3 integrins by SDF-1/CXC12 increases the aggressiveness of prostate cancer cells. Prostate. 2007; 67(1):61-73.

22. Kiel MJ, Morrison SJ. Uncertainty in the niches that maintain haematopoietic stem cells. Nat Rev Immunol. 2008;8(4):290-301.

23. Jung Y, et al. Hematopoietic stem cells regulate mesenchymal stromal cell induction into osteoblasts thereby participating in the formation of the stem cell niche. Stem Cells. 2008;26(8):2042-2051.

24. Jung Y, et al. Annexin II expressed by osteoblasts and endothelial cells regulates stem cell adhesion, homing, and engraftment following transplantation. Blood. 2007;110(1):82-90.

25. Petit I, et al. G-CSF induces stem cell mobilization by decreasing bone marrow SDF-1 and up-regulating CXCR4. Nat Immunol. 2002;3(7):687-694.

26. Kollet O, et al. Osteoclasts degrade endosteal components and promote mobilization of hematopoietic progenitor cells. Nat Med. 2006;12(6):657-664.

27. Hirbe AC, et al. Granulocyte colony-stimulating factor enhances bone tumor growth in mice in an osteoclast-dependent manner. Blood. 2007; 109(8):3424-3431.

28. Park IK, et al. Bmi-1 is required for maintenance of adult self-renewing haematopoietic stem cells. 
Nature. 2003;423(6937):302-305.

29. Iwama A, et al. Enhanced self-renewal of hematopoietic stem cells mediated by the polycomb gene product Bmi-1. Immunity. 2004;21(6):843-851.

30. Weiss L. Metastatic inefficiency. Adv Cancer Res. 1990; 54:159-211.

31. Chambers AF, Naumov GN, Vantyghem SA, Tuck $\mathrm{AB}$. Molecular biology of breast cancer metastasis. Clinical implications of experimental studies on metastatic inefficiency. Breast Cancer Res. 2000. 2(6):400-407.

32. Pantel K, Cote RJ, Fodstad O. Detection and clinical importance of micrometastatic disease. J Natl Cancer Inst. 1999;91(13):1113-1124.

33. Al-Hajj M, Wicha MS, Benito-HernandezA, Morrison
SJ, Clarke MF. Prospective identification of tumorigenic breast cancer cells. Proc Natl Acad Sci U S A. 2003;100(7):3983-3988.

34. Clarke MF, Fuller M. Stem cells and cancer: two faces of eve. Cell. 2006;124(6):1111-1115.

35. Raaijmakers $\mathrm{MH}$, et al. Bone progenitor dysfunction induces myelodysplasia and secondary leukaemia. Nature. 2010;464(7290):852-857.

36. Shiozawa Y, Havens AM, Pienta KJ, Taichman RS The bone marrow niche: habitat to hematopoietic and mesenchymal stem cells, and unwitting host to molecular parasites. Leukemia. 2008;22(5):941-950.

37. Bhattacharya D, Czechowicz A, Ooi AG, Rossi DJ, Bryder D, Weissman IL. Niche recycling through division-independent egress of hematopoietic stem cells. J Exp Med. 2009;206(12):2837-2850.

38. Taichman RS, Loberg RD, Mehra R, Pienta KJ. The evolving biology and treatment of prostate cancer. J Clin Invest. 2007;117(9):2351-2361.

39. Paget $S$. The distribution of secondary growths in cancer of the breast. Lancet. 1889;1:571-573.

40. Wu TT, et al. Establishing human prostate cancer cell xenografts in bone: induction of osteoblastic reaction by prostate-specific antigen-producing tumors in athymic and SCID/bg mice using LNCaP and lineage-derived metastatic sublines. Int J Cancer. 1998;77(6):887-894.

41. Koh AJ, et al. Cells of the osteoclast lineage as mediators of the anabolic actions of parathyroid hormone in bone. Endocrinology. 2005;146(11):4584-4596. 